This item was submitted to Loughborough's Research Repository by the author.

Items in Figshare are protected by copyright, with all rights reserved, unless otherwise indicated.

\title{
Tyre/road interaction noise-A 3D viscoelastic multilayer model of a tyre belt
}

\section{PLEASE CITE THE PUBLISHED VERSION}

http://dx.doi.org/10.1016/j.jsv.2008.09.021

\section{PUBLISHER}

(c) Elsevier Ltd.

\section{VERSION}

SMUR (Submitted Manuscript Under Review)

\section{LICENCE}

CC BY-NC-ND 4.0

\section{REPOSITORY RECORD}

O'Boy, D.J., and A.P. Dowling. 2012. "Tyre/road Interaction Noise-a 3D Viscoelastic Multilayer Model of a Tyre Belt”. figshare. https://hdl.handle.net/2134/9959. 
This item was submitted to Loughborough's Institutional Repository (https://dspace.lboro.ac.uk/) by the author and is made available under the following Creative Commons Licence conditions.

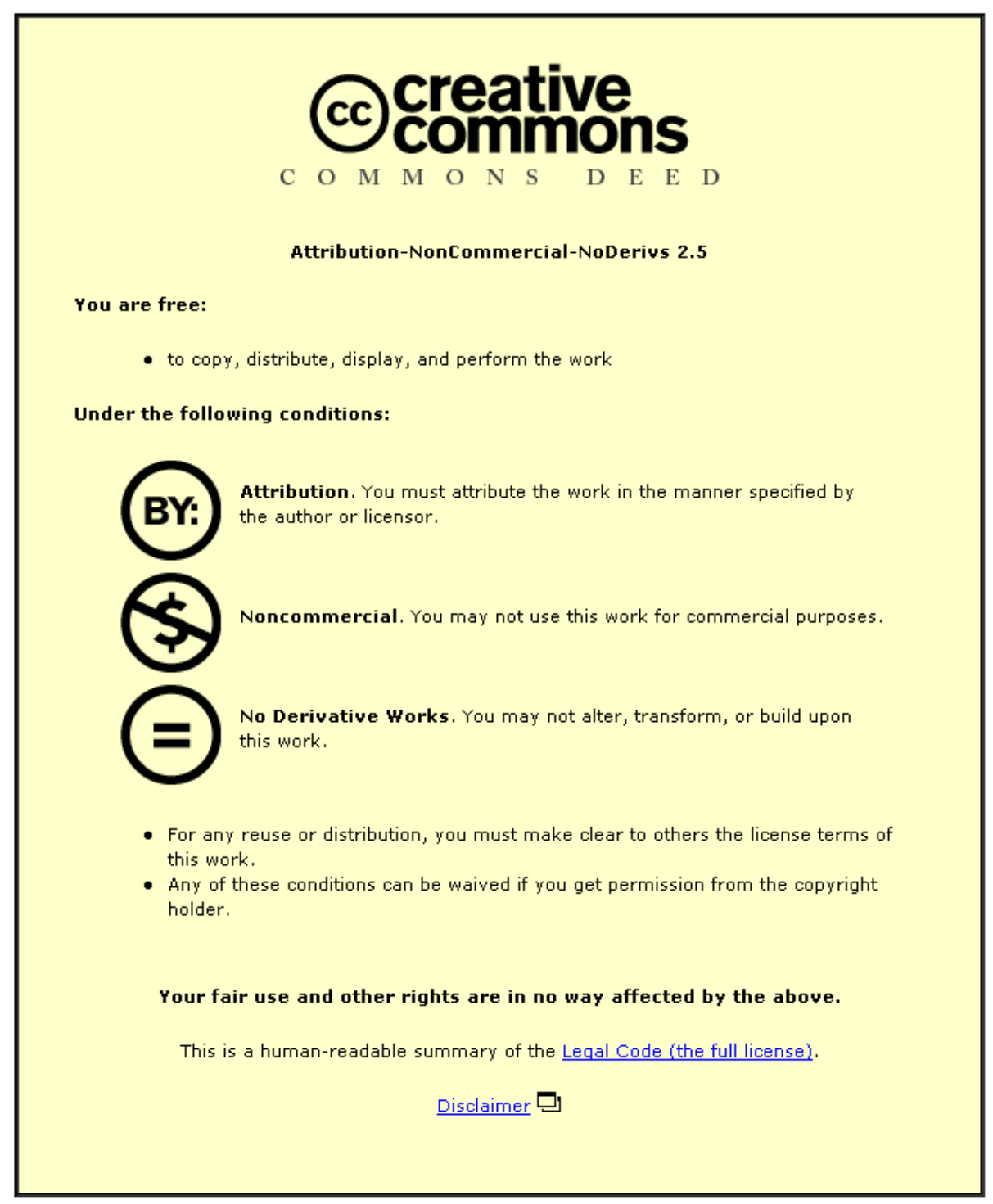

For the full text of this licence, please go to: http://creativecommons.org/licenses/by-nc-nd/2.5/ 


\title{
Tyre / Road Interaction Noise - A 3D viscoelastic multilayer model of a tyre belt
}

\author{
D.J. O'Boy*, A.P.Dowling \\ Department of Engineering, University of Cambridge
}

\begin{abstract}
Vehicle noise is an increasing local environmental problem. For cars, above a steady speed of $40 \mathrm{~km} / \mathrm{hr}$ the noise produced by the interaction of the tyres with the road surface is the dominant noise source.

In order to be able to predict this noise, the vibration characteristics of a stationary tyre must be determined. A multilayer viscoelastic cylindrical representation of the tyre belt, located between the sidewalls of the tyre and excluding the tread, is provided which yields the displacement and velocity response of the tyre belt when excited in the radial or tangential directions for a wide range of excitation frequencies, using only data from the design process.

This model includes a representation of an air cavity and sidewalls and the response of the tyre belt is determined in both the frequency-wavenumber and timespatial domains. The model can then be used to determine the noise of a tyre rolling on a rough road.
\end{abstract}

Key words: Tyre, noise, vibration, viscoelastic multilayer structure, road surface 


\section{Introduction}

One of the main local environmental problems frequently cited in Europe is the noise which is generated by traffic, industrial processes and recreational activities [1]. The reduction in noise from individual cars and trucks since 1970 $(8 \mathrm{~dB}(\mathrm{~A})$ and $11 \mathrm{~dB}(\mathrm{~A})$ respectively) has been negated through the growth in the number of cars on the roads and the longer distances travelled. UK Government forecasts for the future traffic growth in the period 2000-2010 are around 17 percent, a trend which is reflected across Europe [2].

The sources of noise from a vehicle can be separated into four main areas, the engine, exhaust exit, air intake and the noise produced as a result of the tyres rolling in contact with the road surface. Reductions in the levels of noise from the former sources have been significant and it is now recognised that the tyre / road interaction noise is dominant for constant speeds in almost all types of driving [3], certainly above $40 \mathrm{~km} / \mathrm{hr}$ for cars. It is also a significant contributor to the overall noise during acceleration, and is the dominant noise source above a vehicle speed of $50 \mathrm{~km} / \mathrm{hr}$.

In order to be able to predict the noise emitted by a tyre rolling on a rough road surface, detailed information must be known about the vibration characteristics of the tyre when excited by radial and tangential forces. These characteristics include the different waves propagating in the tyre (and their speed), the amplitude of vibration at particular frequencies and the sensitivity

\footnotetext{
* Corresponding author. Department of Engineering, University of Cambridge, Trumpington Street, Cambridge, CB2 1PZ

Email address: djo31@cam.ac.uk (D.J. O'Boy).

URL: http://www.eng.cam.ac.uk/ 〜jo31 (D.J. O'Boy).
} 
of the vibration to changes in the geometry of the tyre.

In this paper, we describe a new alternative model for tyre vibrations which uses information available during the design stage. A mathematical model is introduced of the tyre belt, located between the sidewalls and excluding the tread rubber, which is made up of several thin orthotropic layers of different materials of varying thicknesses laid on each other, with their lines of symmetry at different angles. The tyre is then modelled as an infinitely long periodic cylinder comprised of these various layers, taking into account the significance of individual layers and their curvature. Many existing models are based on assumptions of thin structures, which break down at high frequencies (of interest to exterior noise radiation) where the shortest wavelength in the tyre becomes comparable to the thickness. At these high frequencies, resonances can occur across the tread layers and should therefore be included in any prediction model. This proposed model uses the three-dimensional viscoelastic stress-strain relationships to find the displacement and velocity response of the tyre belt to an applied load in the radial and circumferential directions.

Some previous work has been based on finite element models of the tyre, which are best suited to analysis of lower frequency ranges, or for use at single frequencies of interest due to computational demands, see for example the work of Takahashi [4], Saigal [5] or Pietrzyk [6]. Other mathematical models have determined the vibration of the tyre belt using simplifications to obtain shell, ring and flat bending plate models such as the one developed by Kropp [7][8]. These represent the tyre belt (tyre rubber, steel laminate and textile fibre layer) as a single thin plate with anisotropic material properties derived empirically based on measured response functions (see for example [9] [10] [11] [12] [13]) as analytical methods to determine the parameters require 
strict assumptions and are not usually applicable for the commercial range of materials found in tyres without significant error. Not surprisingly, these produce good agreement for comparisons between the experimental tyre and the model, but this does not provide a predictive capability to model different tyres. Investigations into new belt materials and/or different material thicknesses would potentially need a new experimental tyre belt to be constructed and tested each time to accurately identify these parameters. However, they do allow a simple method to calculate the dynamic properties of a tyre. The proposed model in this paper can include the material properties of each layer, which may be given without experimental validation for a particular tyre belt package.

The model of the tyre belt presented in this paper determines the mechanical response of a complex multi-layered belt and enables the effect of varying the viscoelastic parameters to be investigated theoretically without any need to build experimental tyres. In this work we determine the response of an infinitely long cylinder of the belt material with the aim that it may be used as part of a separate noise prediction program. This further work will utilise the results from this cylindrical model, treating the tyre belt as an idealised problem used to derive the best fit parameters for a simpler bending plate model of the cylinder. This equivalent bending plate may then be used to represent the belt in a finite width tyre model to predict the sound as the tyre rolls on a rough road. Since design data is used as input for the properties of the tyre model, the method developed in this paper enables the effect of different tyre constructions to be predicted and represented without the need to build and test experimental tyres. Although some approximations are still needed, the proposed model can be potentially used for a wide frequency 
range, even at very high frequencies where the finite element analysis becomes impractical. By including details of each layer separately, it is possible to identify a dominant layer for a resonance in the thickness.

The mathematical model of the tyre belt is developed in two stages. With reference to Fig. 1, the viscoelastic belt structure is defined in Section 2, consisting of multiple layers of viscoelastic material, each layer isotropic and infinite in the axial direction, and the form of the general solution is determined. In Section 3 the boundary conditions are specified. They represent an air cavity on the inside surface of the tyre belt and the application of the excitation force on the outer surface. The sidewall stiffness is modelled as a distributed spring force across the width of the tyre, as "Winkler foundation" as in other models and can be determined either analytically or from experimental measurements. An analysis is carried out to identify the waves present and their propagation speeds and results for the tyre belt response in both the wavenumber-frequency and space-time domains are calculated.

\section{Multilayer 3D viscoelastic tyre belt model}

The governing equation for a layer of homogeneous isotropic material is developed by considering a volume element with applied stresses. The strain in the layer may be expressed using index notation with the summation convention as $\epsilon_{i j}=\frac{1}{2}\left(u_{i, j}+u_{j, i}\right)$ where $u$ is the displacement and $u_{i, j}=\partial u_{i} / \partial x_{j}$. In an isotropic elastic material, Hooke's law relates the stress to the strain through

the material properties (Lamé coefficients), $\sigma_{i j}=\lambda \delta_{i j} \epsilon_{k k}+2 \mu \epsilon_{i j}$, where $\delta_{i j}$ denotes the Kronecker delta $\left(\delta_{i j}=1\right.$ for $i=j$ and $\delta_{i j}=0$ for $\left.i \neq j\right)[14]$. 
The Lamé coefficients may be written in terms of the Young's modulus of the layer $E$, Poisson's ratio $\nu$ and the mass per unit volume of the material $\rho$. Then $\lambda=E \nu /((1+\nu)(1-2 \nu))$ and the shear modulus $\mu=E / 2(1+\nu)$. Any model of the tyre must take into account the energy lost when an element of material distorts and kinetic energy is converted into heat. This damping is introduced by allowing a complex Young's modulus. Substitution of the expressions for stress and strain into Newton's second law yields the equation of motion in vector notion, where $\nabla$ denotes the vector operator, $\left(\partial / \partial x_{1}, \partial / \partial x_{2}, \partial / \partial x_{3}\right)$ and $\ddot{\mathbf{u}}$ is the double differential of the vector $\mathbf{u}$ with respect to time.

$$
(\lambda+\mu) \nabla(\nabla \cdot \mathbf{u})+\mu \nabla^{2} \mathbf{u}=\rho \ddot{\mathbf{u}}
$$

In order to obtain two separate wave equations, the displacement vector is decomposed into the sum of a scalar $\phi$ and vector potential $\mathbf{A}$, from a theorem by Helmholtz [15]. This approach is consistent with the methodology used by Gazis [16] when examining the free vibrations of cylinders and will therefore be applicable in cylindrical coordinates to construct a mathematical model of the tyre belt. The two separate wave equations are obtained when the divergence of the vector potential equals zero.

$$
\mathbf{u}=\nabla \phi+\nabla \times \mathbf{A}, \quad \nabla \cdot \mathbf{A}=0 .
$$

Using a set of five vector identities [17], it may be shown that Eq. (1) can be decoupled into two wave equations, the first referring to a wave which causes a change in volume of the material, propagating with speed $\alpha$ and the second causing a rotational disturbance with no change in volume, propagating at a speed $\beta$ (where the term $\ddot{\phi}$ represents the double differentiation of $\phi$ with respect to time), 


$$
\begin{aligned}
\nabla^{2} \phi & =\frac{1}{\alpha^{2}} \ddot{\phi}, & \alpha^{2}=\left(\frac{\lambda+2 \mu}{\rho}\right), \\
\nabla^{2} \mathbf{A} & =\frac{1}{\beta^{2}} \ddot{\mathbf{A}}, & \beta^{2}=\left(\frac{\mu}{\rho}\right) .
\end{aligned}
$$

At this point, an infinitely long isotropic viscoelastic cylindrical layer is defined, with the aim of characterising the belt properties by determining the relationship between the traction and displacement on the inner and outer radial surfaces of the layer. It is convenient to introduce Fourier transforms which relate the cylindrical polar coordinates $(r, t, \theta, z)$, where $t$ is time, to $\left(r, \omega, n, k_{z}\right)$ as shown in Eq. (5) [The use of $\tilde{u}$ indicates the Fourier transform has been applied to the variable $u$ ]. In the Fourier domain $n$ is the angular order, $k_{z}$ the axial wavenumber and $\omega$ the frequency. Eq. (5) may be inverted to give Eq. (6),

$$
\begin{aligned}
\tilde{u}\left(r, \omega, n, k_{z}\right) & =\int_{-\infty}^{\infty} \int_{-\infty}^{\infty} \int_{-\pi}^{\pi} u(r, \theta, z, t) e^{-\mathrm{i} n \theta} e^{-\mathrm{i} k_{z} z} e^{-\mathrm{i} \omega t} \mathrm{~d} \theta \mathrm{d} z \mathrm{~d} t \\
u(r, t, \theta, z) & =\frac{1}{(2 \pi)^{3}} \int_{-\infty}^{\infty} \int_{-\infty}^{\infty} \sum_{n=-\infty}^{\infty} \tilde{u}\left(r, n, k_{z}, \omega\right) e^{\mathrm{i} n \theta} e^{\mathrm{i} k_{z} z} e^{\mathrm{i} \omega t} \mathrm{~d} k_{z} \mathrm{~d} \omega .
\end{aligned}
$$

The Fourier transform, Eq. (5), is applied to the wave equations to obtain radial solutions to the scalar and vector potentials. The application of Eq. (5) to the scalar wave equation, Eq. (3), yields

$$
\frac{\partial^{2} \tilde{\phi}}{\partial r^{2}}+\frac{1}{r} \frac{\partial \tilde{\phi}}{\partial r}-\frac{n^{2}}{r^{2}} \tilde{\phi}-k_{z}^{2} \tilde{\phi}=-\frac{\omega^{2}}{\alpha^{2}} \tilde{\phi}
$$

Defining $\gamma^{2}=\left(\omega^{2} / \alpha^{2}-k_{z}^{2}\right)$ allows a rearrangement of this expression into the form of a Bessel equation [18] which has a solution with two parts, composed of Bessel functions of the first and second kind,

$$
\tilde{\phi}(\gamma r)=c_{1} J_{n}(\gamma r)+c_{2} Y_{n}(\gamma r)
$$

where $c_{1}\left(\omega, n, k_{z}\right)$ and $c_{2}\left(\omega, n, k_{z}\right)$ are constants to be determined through the 
application of boundary conditions for stress and strain. A special case of solution exists for a viscoelastic solid rod with no inner radial surface. Then the solution is required to be regular at the centre $r=0 \mathrm{~m}$, where the second Bessel function is singular, $Y_{n}(0)$. The solution is obtained by setting the second constant equal to zero, leaving the solution to the scalar wave equation as, $\tilde{\phi}(\gamma r)=c_{1} J_{n}(\gamma r)$.

Solutions are also required for all components of the vector potential A. Since the axial term of this vector $A_{z}$ is decoupled from the two other components, it may be shown that the radial solution after taking Fourier transforms is similar to Eq. (8), with constants $c_{7}\left(\omega, n, k_{z}\right)$ and $c_{8}\left(\omega, n, k_{z}\right)$ to be determined through the application of boundary conditions. The term $\eta^{2}=\left(\omega^{2} / \beta^{2}-k_{z}^{2}\right)$.

$$
\tilde{A}_{z}=c_{7} J_{n}(\eta r)+c_{8} Y_{n}(\eta r)
$$

The two final vector components $A_{r}$ and $A_{\theta}$ are coupled in cylindrical coordinates, however it may be shown [17] that these may also be expressed in terms of Bessel functions,

$$
\begin{aligned}
& \left(\mathrm{i} \tilde{A}_{r}-\tilde{A}_{\theta}\right)=2 c_{3} J_{(n+1)}(\eta r)+2 c_{4} Y_{(n+1)}(\eta r), \\
& \left(\mathrm{i} \tilde{A}_{r}+\tilde{A}_{\theta}\right)=2 c_{5} J_{(n-1)}(\eta r)+2 c_{6} Y_{(n-1)}(\eta r) .
\end{aligned}
$$

These two equations may now be solved simultaneously and rearranged using the recurrence relation to be expressed as functions of angular order $n$ and $n+1$ only, which minimises numerical calculations [see [17], Eq. (2.29)]. The solutions to the vector potential contain six constants, however it is possible to further eliminate two constants $\left(c_{5}\right.$ and $c_{6}$ are chosen as the most suitable for elimination) from the previous equations by employing the condition of zero divergence on the vector potential stated in Eq. (2). 
This differs from the approach taken by Gazis [16] [19], who chooses to eliminate two constants by assuming that $A_{r}(r, t)=-A_{\theta}(r, t)$. Then the divergence of the vector potential is non-zero and he wrongly assumes it is still possible to obtain two separate wave equations. The result of this incorrect assumption being that important coupling between the radial, angular and axial potentials is neglected, which becomes apparent when frequency and both angular and axial wavenumbers are non-zero. Applying the Fourier transform to the expression for the divergence of the vector potential yields,

$$
\nabla \cdot \tilde{\mathbf{A}}=\frac{\partial \tilde{A}_{r}}{\partial r}+\frac{1}{r} \tilde{A}_{r}+\frac{\mathrm{i} n}{r} \tilde{A}_{\theta}+\mathrm{i} k_{z} \tilde{A}_{z}=0
$$

The solutions for the components of the vector potential, Eqs. (9-11) are substituted into Eq. (12) and rearranged (this step includes a differentiation with respect to the radius using the chain rule). Once the manipulation is concluded, we obtain Eq. (13), for which each group of terms in the brackets must reduce to zero as solutions are required for all angular orders and radii (each group must be independent of the other, whether the cylinder is solid or hollow).

$$
\begin{gathered}
0=J_{n}(\eta r)\left\{-c_{3} \eta+c_{5} \eta+c_{7} k_{z}\right\}+Y_{n}(\eta r)\left\{-c_{4} \eta+c_{6} \eta+c_{8} k_{z}\right\}, \\
c_{5}=\left(c_{3} \eta-k_{z} c_{7}\right) / \eta, \quad c_{6}=\left(c_{4} \eta-k_{z} c_{8}\right) / \eta .
\end{gathered}
$$

The three components of displacement in the viscoelastic layer were originally expressed as a Helmholtz decomposition of two potentials, provided in Eqs. (A.1-A.3) of the appendix. Since solutions to these potentials have now been derived in terms of Bessel functions, it is now possible to write the components of displacement in terms of the six constants. The stress in the layer is related to the derivatives of the displacements, so these may also be written in the 
same format.

When the radial solutions to the scalar and vector potentials are substituted into Eqs. (A.1-A.3), the components of displacement may then be written in terms of a six by three matrix $\mathbf{h}$ and the as yet unknown coefficients,

$$
\left(\tilde{u}_{r}, \tilde{u}_{\theta}, \tilde{u}_{z}\right)^{\mathrm{T}}=\mathbf{h}\left(c_{1}, c_{2}, c_{3}, c_{4}, c_{7}, c_{8}\right)^{\mathrm{T}}
$$

The matrix $\mathbf{h}$ is a function of the radius $r$, wavenumbers $n, k_{z}$, the frequency $\omega$ and the material properties of the layer.

In a similar way, the three stresses which lead to traction on a cylindrical surface, whose normal is in the radial direction, are Fourier transformed to give Eqs. (A.4-A.5) in the appendix, which may then be written as a function of the six constants through a six by three matrix $\mathbf{g}$,

$$
\left(\tilde{\sigma}_{r r}, \tilde{\sigma}_{r \theta}, \tilde{\sigma}_{r z}\right)^{\mathrm{T}}=\mathbf{g}\left(c_{1}, c_{2}, c_{3}, c_{4}, c_{7}, c_{8}\right)^{\mathrm{T}}
$$

The matrices $\mathbf{g}$ and $\mathbf{h}$ contain all information on how the material properties in the viscoelastic layer affect the stresses and displacements, for all wavenumbers and frequencies. It should be noted that both matrices are dependent on the radial position $r$.

The inclusion of Bessel functions in the form of the solutions for the cylindrical geometry adds additional numerical problems over the exponential functions found in a Cartesian coordinate system for plane layers. It is therefore advantageous to split the matrices $\mathbf{g}$ and $\mathbf{h}$ into several symmetric matrices, with components which are common and may be inverted with ease. Through manipulation of the six by six matrix $(\mathbf{g}, \mathbf{h})^{\mathrm{T}}$, the components of stress and displacement on the lower surface of layer $j$ may be related to the coefficients 
within that layer. The relationship is written in the form

$$
\left(\begin{array}{c}
\tilde{\boldsymbol{\sigma}} \\
\tilde{\mathbf{u}}
\end{array}\right)_{r_{j}}=\left(\begin{array}{c}
\mathbf{G} \\
\mathbf{H}
\end{array}\right)_{r_{j}} \mathbf{W}_{r_{j}} \mathbf{S}_{r_{j}} \mathbf{C}_{r_{j}}
$$

$\mathbf{C}$ is a vector of the constants which include one Bessel function for numerical convenience,

$$
\mathbf{C}_{r_{j}}=\left(c_{1} J_{n}(\gamma r), c_{2} Y_{n}(\gamma r), c_{3} J_{n+1}(\eta r), c_{4} Y_{n+1}(\eta r), c_{7} J_{n}(\eta r), c_{8} Y_{n}(\eta r)\right)^{\mathrm{T}}
$$

The full form of $\mathbf{G}, \mathbf{H}, \mathbf{W}$ and $\mathbf{S}$ are given in the appendix (Eqns. A.15 - A.52). W has only diagonal terms which depend on the Wronskian expressions for the Bessel functions and $\mathbf{S}$ is a summation matrix.

The components of stress and displacement on the outer surface of layer $j$ may also be written in a similar form, as

$$
\left(\begin{array}{c}
\tilde{\boldsymbol{\sigma}} \\
\tilde{\mathbf{u}}
\end{array}\right)_{r_{j+1}}=\left(\begin{array}{c}
\mathbf{G} \\
\mathbf{H}
\end{array}\right)_{r_{j+1}} \mathbf{W}_{r_{j+1}} \mathbf{S}_{r_{j+1}} \mathbf{C}_{r_{j+1}}
$$

Inspection shows that $\mathbf{C}_{r_{j+1}}=\mathbf{M} \mathbf{C}_{r_{j}}$ where the matrix $\mathbf{M}$ acts as a scaling factor dependent on the thickness of the layer. Substitution of Eq. (17) into Eq. (19) yields an expression relating the components of stress and displacement on one face of the elastic layer to the components on the other face, using an overall material matrix $\mathbf{X}_{j}$, which is a function of frequency, wavenumber, material properties of the layer its inner and outer radii.

$$
\left(\begin{array}{c}
\tilde{\boldsymbol{\sigma}} \\
\tilde{\mathbf{u}}
\end{array}\right)_{r_{j+1}}=\left(\begin{array}{c}
\mathbf{G} \\
\mathbf{H}
\end{array}\right)_{r_{j+1}} \mathbf{W}_{r_{j+1}} \mathbf{S} \mathbf{M}_{r_{j+1, j}} \mathbf{S}^{-1} \mathbf{W}_{r_{j}}^{-1}\left(\begin{array}{l}
\mathbf{G} \\
\mathbf{H}
\end{array}\right)_{r_{j}}^{-1}\left(\begin{array}{c}
\tilde{\boldsymbol{\sigma}} \\
\tilde{\mathbf{u}}
\end{array}\right)_{r_{j}}
$$




$$
\mathbf{X}_{j}=\left(\begin{array}{l}
\mathbf{G} \\
\mathbf{H}
\end{array}\right)_{r_{j+1}} \mathbf{W}_{r_{j+1}} \mathbf{S} \mathbf{M}_{r_{j+1, j}} \mathbf{S}^{-1} \mathbf{W}_{r_{j}}^{-1}\left(\begin{array}{l}
\mathbf{G} \\
\mathbf{H}
\end{array}\right)_{r_{j}}^{-1} .
$$

This overall material matrix relates the components and stress on one surface of a single viscoelastic cylindrical layer to those components on the other surface.

$$
\left(\begin{array}{c}
\tilde{\boldsymbol{\sigma}} \\
\tilde{\mathbf{u}}
\end{array}\right)_{r_{j+1}}=\mathbf{X}_{j}\left(\begin{array}{c}
\tilde{\boldsymbol{\sigma}} \\
\tilde{\mathbf{u}}
\end{array}\right)_{r_{j}} .
$$

The tyre belt is made up of different layers of material with the outermost layer in contact with the atmosphere through the tread blocks and the innermost layer in contact with an air cavity, with high air pressure sealed by the sidewalls and wheel hub. To model the tyre belt accurately, these multiple layers of material must be taken into account as shown in Fig. 2 which illustrates a multilayer cylindrical surface, in which each layer is defined by the density, Young's modulus, Poisson's ratio and damping (through the complex Young's modulus). The thickness of each individual layer is known, allowing one overall material matrix to be determined for the structure (the composite sidewall is not included in this model).

An expression for the components of stress and displacement in layer $j$ is given in Eq. (22). Writing the same expression for the stress and displacement in the layer denoted $j-1$ gives,

$$
\left(\begin{array}{c}
\tilde{\boldsymbol{\sigma}} \\
\tilde{\mathbf{u}}
\end{array}\right)_{r_{j}}=\mathbf{X}_{j-1}\left(\begin{array}{l}
\tilde{\boldsymbol{\sigma}} \\
\tilde{\mathbf{u}}
\end{array}\right)_{r_{j-1}}
$$

In order to develop an expression covering multiple layers of material, it is 
assumed that when two layers of material are in contact, there is no slip between them and contact is maintained at all times. It is then valid to suggest that traction and displacement are continuous at the interface between layers. If $(\tilde{\boldsymbol{\sigma}}, \tilde{\mathbf{u}})^{\mathrm{T}}$ are the same in the two layers which meet at $r=r_{j}$, then Eq. (23) can be substituted into Eq. (22) giving,

$$
\left(\begin{array}{c}
\tilde{\boldsymbol{\sigma}} \\
\tilde{\mathbf{u}}
\end{array}\right)_{r_{j+1}}=\mathbf{X}_{j} \mathbf{X}_{j-1}\left(\begin{array}{c}
\tilde{\boldsymbol{\sigma}} \\
\tilde{\mathbf{u}}
\end{array}\right)_{r_{j-1}} .
$$

Repeating this substitution for all layers yields an overall matrix linking the traction and displacement on the outer layer of the tyre to that on the most inner layer, as shown in Fig. 2, with two main advantages. The first is that when boundary conditions are applied to the surfaces, it will be the most outer and inner surfaces for which information will be available and the second is that this approach yields one overall material matrix which implicitly contains all of the design data on each layer of the tyre.

$$
\left(\begin{array}{c}
\tilde{\boldsymbol{\sigma}} \\
\tilde{\mathbf{u}}
\end{array}\right)_{r_{N+1}}^{N} \prod_{j=1} \mathbf{X}_{j}\left(\begin{array}{c}
\tilde{\boldsymbol{\sigma}} \\
\tilde{\mathbf{u}}
\end{array}\right)_{r_{1}}
$$

The form of these equations are valid for almost all values of frequency, angular order and axial wavenumbers, however the case of the displacement of a surface for a steady state force applied uniformly is an exception, with solutions obtained from Timoshenko [20], who examined the case of a stress distribution which is symmetrical about an axis using work from Lamé [21]. 


\section{Boundary conditions applied to the tyre belt}

In order to solve the Eq. (25) and determine the displacement response to a force input in the Fourier domain, six boundary conditions must be supplied. For the inner belt surface, these boundary conditions include a mathematical approximation of an air cavity under pressure and for the outer surface, it is assumed that the belt is exposed to the atmosphere with an imposed localised force.

The forces on the inside surface of the tyre belt are generated by the air pressure bounded by the wheel hub, tyre belt and sidewalls, which also apply a supporting force on the edges of the belt. A cross section of the wheel hub and air cavity is shown in Fig. 3 , with mean pressure $p_{0}$ and density $\rho_{0}$. The following section describes the relationship between the components of stress and displacement on the inner surface $(\tilde{\boldsymbol{\sigma}}, \tilde{\mathbf{u}})_{r=r_{2}}^{\mathrm{T}}$, specifically the radial boundary condition. Two further boundary conditions on the inner surface of the tyre belt come from a condition of zero shear stress on the interface between the air and the tyre belt as the air is effectively inviscid and unable to exert a significant shear stress on the belt.

At the interface between the air and the fixed wheel hub $r=r_{1}$, there is assumed to be no radial displacement, $u_{r}=0 \mathrm{~m}$. The air in the cavity is assumed to be inviscid with pressure and density perturbations $p^{\prime}(r, t, \theta, z)$, $\rho^{\prime}$ respectively, which satisfy a wave equation with sound speed (pressurised air inside the tyre cavity) $c_{c}, \nabla^{2} p^{\prime}=\ddot{p}^{\prime} / c_{c}^{2}$. Substituting for $\nabla^{2}$ in cylindrical coordinates and applying the Fourier transform yields an equation in the form 
of Bessel's differential equation,

$$
\begin{gathered}
\frac{\partial^{2} \tilde{p}^{\prime}}{\partial r^{2}}+\frac{1}{r} \frac{\partial \tilde{p}^{\prime}}{\partial r}+\left(\Gamma^{2}-\frac{n^{2}}{r^{2}}\right) \tilde{p}^{\prime}=0 \\
\tilde{p}^{\prime}=C_{a 1} J_{n}(\Gamma r)+C_{a 2} Y_{n}(\Gamma r)
\end{gathered}
$$

where $c_{c}$ is the speed of sound in the air cavity, $C_{a 1}$ and $C_{a 2}$ are constants and $\Gamma=\sqrt{\omega^{2} / c_{c}^{2}-k_{z}^{2}}$.

The Fourier transform of the radial component of the momentum equation, $\tilde{u}_{r}=\left(1 / \rho_{0} \omega^{2}\right) \partial \tilde{p}^{\prime} / \partial r$, is used to obtain a relation between the pressure perturbation and the displacement in the gas. After substituting the derivative of the pressure in the radial direction using Eq. (27), the Fourier transform of the displacement is found as a function of the gas properties, the frequency and wavenumbers,

$$
\tilde{u}_{r}=\frac{C_{a 1}}{\rho_{0} \omega^{2}}\left(\frac{n}{r} J_{n}(\Gamma r)-\Gamma J_{n+1}(\Gamma r)\right)+\frac{C_{a 2}}{\rho_{0} \omega^{2}}\left(\frac{n}{r} Y_{n}(\Gamma r)-\Gamma Y_{n+1}(\Gamma r)\right)
$$

This equation may be further simplified by elimination of one of the constants, using the assumption that at the wheel hub, $r=r_{h}$, the radial displacement of the gas is zero. This allows the relationship between the two constants to be written $C_{a 2}=A_{\text {air }} C_{a 1}$, where $A_{\text {air }}$ is a scaling factor between the two.

$$
A_{\text {air }}=-\left(\frac{n}{r_{h}} J_{n}\left(\Gamma r_{h}\right)-\Gamma J_{n+1}\left(\Gamma r_{h}\right)\right) /\left(\frac{n}{r_{h}} Y_{n}\left(\Gamma r_{h}\right)-\Gamma Y_{n+1}\left(\Gamma r_{h}\right)\right) .
$$

It will be assumed that at the interface between the air and the tyre belt rubber, $r=r_{2}$, there is continuity of stress and displacement, such that $\left(\tilde{p}^{\prime}, \tilde{u}_{r}\right)_{\left(r=r_{2}\right)}^{\mathrm{T}}=\left(-\tilde{\sigma}_{r r}, \tilde{u}_{r}\right)_{\left(r=r_{2}\right)}^{\mathrm{T}}$. Substituting Eq. (29) into (28) and evaluating at the radius $r=r_{2}$ yields,

$$
\begin{aligned}
\tilde{p}_{\left(r=r_{2}\right)} & =\left(J_{n}\left(\Gamma r_{2}\right)+A_{\mathrm{air}} Y_{n}\left(\Gamma r_{2}\right)\right) C_{a 1}, \\
\tilde{u}_{r\left(r=r_{2}\right)} & =B_{\mathrm{air}} C_{a 1},
\end{aligned}
$$


where the term $B_{\text {air }}$ is given by,

$$
B_{\text {air }}=\frac{1}{\rho_{0} \omega^{2}}\left(\frac{n}{r_{2}} J_{n}\left(\Gamma r_{2}\right)-\Gamma J_{n+1}\left(\Gamma r_{2}\right)\right)+\frac{A_{\text {air }}}{\rho_{0} \omega^{2}}\left(\frac{n}{r_{2}} Y_{n}\left(\Gamma r_{2}\right)-\Gamma Y_{n+1}\left(\Gamma r_{2}\right)\right) .
$$

Since the two equations for pressure perturbation and radial displacement at the interface are functions of a single unknown $C_{a 1}$, they can be related by introducing an effective air spring stiffness defined as, $\tilde{p} / \tilde{u}_{r\left(r=r_{2}\right)}=\left(J_{n}\left(\Gamma r_{2}\right)+\right.$ $\left.A_{\text {air }} Y_{n}\left(\Gamma r_{2}\right)\right) / B_{\text {air }}$

This effective air stiffness is shown in Fig. 4 for a tyre with a hub radius of $r=0.22 \mathrm{~m}$ and a gauge inflation pressure of $1.9 \mathrm{bar}$, for periodic boundary conditions in the axial directions, $k_{z}=m \pi / w$, where $w=0.18 \mathrm{~m}$ as the width of the tyre and $m$ is the axial mode number $m=0,1,2,3$. This is idealised as in reality the sidewalls will be able to deform in the radial and axial directions, however, this simplification assumes that the resistive force from the sidewalls is distributed over the width of the tyre. The accurate modelling of the sidewall force is most important when examining the very low frequency vibrations which lead to noise transmission to the interior of the vehicle rather than for the much higher acoustic frequencies for exterior noise. The pinned sidewall assumption can be used to find the point mobility response at the centre of the free standing tyre, to obtain information on the response of the damped multilayer cylindrical structure. This is useful for design as it allows each layer of the belt structure to be modified at a time, either for thickness, stiffness or damping while keeping the other tyre parameters constant.

Further work could be performed to incorporate the sidewalls with a more accurate representation, utilising forces and moments at the tyre edges, however these characterisations would be for specific manufacturing methods, tyre de- 
signs and geometries and could be included with mathematics to include tyre rotation and a ground contact point.

It may be seen that there are several resonances in the air cavity which provide high pressures for small displacements, such that the belt is effectively against a very stiff boundary. For these resonances, the rubber in the belt and tread will be excited with an amplified compression mode. However, the air cavity will only be of importance for high frequencies and/or low angular orders, $n<10$ if the aim of the model is accounting for vibrations in the acoustic frequency range $f=0-3 \mathrm{kHz}$.

As the pressure of the air inside the tyre cavity is increased from atmospheric to a higher pressure, the tyre radius increases. To prevent the structure of the tyre breaking apart, bands of stiff material are included in the tyre belt, wrapped around circumferentially and axially. Although the primary aim of these bands is to maintain the structure of the tyre with high steady state internal pressure and centrifugal acceleration, they also provide a resistive force to radial motion, similar to a spring.

It is assumed that such a band can be modelled by a cylindrical membrane with tension $\bar{T}$, at a radius $r=r_{b}$ from the centre of the tyre. The steady state stress in the radial direction on the inside of the band is $\bar{\sigma}_{r r \text { (in) }}$ and the radial stress on the outside $\bar{\sigma}_{r r(\text { out })}$. The force created by this radial stress in the horizontal direction is the stress multiplied by the projected area and for the case of a surface in equilibrium, a force balance gives $\bar{\sigma}_{r r(\text { in })}=\bar{\sigma}_{r r(\text { out })}+\bar{T} / r_{b}$, assuming unit depth in the axial direction. Using the internal tyre and atmospheric pressures, it is possible to find the tension in the band. Now assuming a stress perturbation on the inside of the band, $\sigma_{r r(\text { in) }}$ and the outside $\sigma_{r r(\text { out })}$, with a 
small change in radius $u_{r}$ causing a change in the band tension $T$, the change in stress across the band may be written as $\sigma_{r r(\text { in })}=\sigma_{r r(\text { out })}-k_{b} u_{r}$, where the band radial stiffness is estimated as $k_{b}=\bar{T} / r_{b}^{2}$. This band stiffness is written using a six by six matrix, $\mathbf{K}_{\text {belt }}$, assuming that the components of unsteady shear stress and the two components of unsteady displacement, $u_{\theta}$ and $u_{z}$, do not change across the band.

$$
\left(\begin{array}{c}
\tilde{\boldsymbol{\sigma}} \\
\tilde{\mathbf{u}}
\end{array}\right)_{r_{b} \text { (outer) }}=\mathbf{K}_{\text {belt }}\left(\begin{array}{c}
\tilde{\boldsymbol{\sigma}} \\
\tilde{\mathbf{u}}
\end{array}\right)_{r_{b} \text { (inner) }}
$$

The advantage of this formulation is that is allows the band to be placed in-between any two layers of the tyre belt. The components of stress and displacement on the inner and outer tyre belt surfaces are still related by an overall matrix representing the material properties and the circumferential bands. As an example, if the band were to be substituted into the model for the belt between layers $j=3$ and $j=4$ it would modify Eq. (25) as follows,

$$
\left(\begin{array}{c}
\tilde{\boldsymbol{\sigma}} \\
\tilde{\mathbf{u}}
\end{array}\right)_{r_{N+1}}=\prod_{j=4}^{N} \mathbf{X}_{j} \mathbf{K}_{\text {belt }} \prod_{j=1}^{3} \mathbf{X}_{j}\left(\begin{array}{c}
\tilde{\boldsymbol{\sigma}} \\
\tilde{\mathbf{u}}
\end{array}\right)_{r_{1}}
$$

The interface between the inside surface of the tyre structure and the air cavity supplies three boundary conditions; it is assumed that the air provides no shear stress $\sigma_{r \theta}, \sigma_{r z}=0$, using the notation for the components of Eq. (34). The third boundary condition is the relationship between the radial stress and radial displacement, due to the assumption that the resistive force provided by the sidewalls distributed over the tyre width together with the gauge inflation pressure can be represented by a distributed spring stiffness $\left(\sigma_{r r} / u_{r}=2 \mathrm{MPa}\right)$. 
Boundary conditions are also applied to the outer surface of the tyre belt. Although the force applied in a direction normal to the surface is the most important to characterise the tyre belt vibration modes and resonant frequencies for vehicles travelling at constant speed over a rough road surface, the components of shear force in the axial and angular directions may also be employed to investigate the response of the tyre when the vehicle is travelling around a corner, accelerating or braking. These conditions are applied for the case where the tyre is non-rotating, surrounded by air and there is no contact with a road surface.

The stress applied to the outer surface may take the form of an impulse (delta function) or a stress distributed over a small area of the outer surface of the tyre belt. The other two stresses are assumed to be zero, which provides six boundary conditions in Eq. (25) and allows the solution to be found, commonly the radial and angular displacement on the outer surface.

\section{Analysis of vibration modes in a thin elastic layer}

The different modes of vibration of a cylindrical shell may be determined using the equations for stress and displacement presented in Eq. (17). By considering simplifications such as vibration independent of axial or angular position, the torsional, longitudinal and flexural modes can be located. The latter modes are determined by locating the roots of the Pochhammer frequency equation, as performed by Gazis for the $n=0$ and $n=1$ family of flexural modes [22]. As the geometry is cylindrical, the solutions of this equation involve Bessel's functions, which add complexity to any analysis. This section compares the modes of vibration found in the proposed model to the waves found in a thin 
plate (for which the equations can be solved relatively quickly), comparing the results for flexural waves and in-plane motion. The comparison can be made for shells where the radius is large and the thickness is thin such that the effect of curvature may be neglected.

A simplified analysis can be undertaken through the use of the equations for a viscoelastic layer in Cartesian coordinates and considering the case of motion independent of axial position, $k_{z}=0 / \mathrm{m}$ with a layer of thickness $y=2 b$ and a wavenumber in the $x$ direction $k_{x}$. The free stress conditions on the surfaces yield the Rayleigh-Lamb frequency equation [22] which may be expressed for symmetric $(A=1)$ or asymmetric modes $(A=-1)$ as,

$$
\frac{\tan (\eta b)}{\tan (\gamma b)}+\left\{\frac{4 \gamma \eta k_{x}^{2}}{\left(k_{x}^{2}-\eta^{2}\right)^{2}}\right\}^{A}=0
$$

If the wavelength is large compared to the thickness of the layer, one solution to this equation occurs for asymmetric modes when $k_{x}>\omega / \alpha, \omega / \beta$, such that the wave speed, $c_{\text {(asym) }}=\omega / k_{x}<\alpha, \beta$. The flexural wave speed in an elastic plate (also applicable as a shear mode), as both the upper and lower surfaces move in phase in the vertical plane, is then

$$
c_{(\text {asym })}=k_{x} b \sqrt{\frac{E}{3 \rho\left(1-\nu^{2}\right)}} .
$$

Another solution to the Rayleigh-Lamb frequency equation occurs when $\omega / \beta>$ $k_{x}>\omega / \alpha$, such that the wave speed, $\beta<c_{(\text {sym })}<\alpha$. The solution exists when the axial displacement $u_{x}$ is symmetric (so the layer is in compression and extension in phase with bulge waves propagating along the layer). This wave speed is given by

$$
c_{(\mathrm{sym})}=\sqrt{\frac{E}{\rho\left(1-\nu^{2}\right)}} .
$$

Due to the assumptions used to determine these speeds, they are only applica- 
ble when the wavelength in the radial direction of the tyre is large compared to the thickness. These wave speeds may be compared to the response of a single viscoelastic cylindrical layer resting on an air cavity, using applied forcing on one surface. In the example shown in Fig. 5, the layer has a thickness of $1.54 \mathrm{~mm}$, which is comparable to the thickness of individual tyre belt layers and has a Young's modulus of $E=4.2 \mathrm{MPa}$, Poisson's ratio $v=0.38$ and density $\rho=1163 \mathrm{kgm}^{-3}$. The ratio of radial displacement to radial forcing $\tilde{u}_{r} / \tilde{\sigma}_{r r}\left(r, \omega, n, k_{z}\right)$ is shown in Fig. 5 (a) for the case of motion independent of axial wavenumber $k_{z}=0 / \mathrm{m}$. It can be seen that the amplitude of the flexural motion dominates the motion due to compression of the material or the resonance from the air cavity (in this thin wall test case, the amplitude of the air cavity resonance is so low that it is only visible for very low angular orders and frequencies in Fig. 5(a)). The mode types are identified in the accompanying plot of Fig. 5(b) for completeness and it should be noted that the speed of the wave in the material may be determined from $c=\omega / k$, where $c$ is the speed and $k$ is the wavenumber.

Also shown is the tangential displacement response to radial forcing, denoted $\tilde{u}_{\theta} / \tilde{\sigma}_{r r}\left(r, \omega, n, k_{z}\right)$ in Fig. 5(c) which has both a significant flexural and compression mode of vibration visible. These tangential vibration modes occur with radial forcing as the material has a Poisson's ratio which tends towards incompressibility, such that a compression in the radial direction is accompanied by an expansion in the tangential direction. As will be shown for the response of the tyre belt, this tangential displacement is minimised by the use of the steel cords in the belt.

The thickness of the single layer is now increased to $10.54 \mathrm{~mm}$, which is large in comparison to the thickness of an individual tyre belt layer and the ratio 
of radial displacement to radial forcing $\tilde{u}_{r} / \tilde{\sigma}_{r r}\left(r, \omega, n, k_{z}\right)$ shown in Fig. 6(a). The flexural wave is still dominant, however the compression of the material is now apparent. As the axial wavenumber increases, the cut off frequencies will increase and the amplitude of the response will decrease. The location of the resonances may be altered by adjusting the material properties, for example, to move the formation of the modes shown in Fig. 6(a) towards lower angular orders would require either a decrease in the density of the material or an increase in the value of Young's modulus, Poisson's ratio or the thickness of the layer. Each of these choices has structural implications, however when considering a multilayer structure, it is possible to reduce the amplitude of the flexural vibration mode by ensuring that no two adjoining layers have similar resonant frequencies.

The variations in radial displacement and stress through the layer for the compression mode, Figs. 6(b) and (c) respectively, are located at a frequency of approximately $1.4 \mathrm{kHz}$ when the angular order $n=7$ and the axial wavenumber is zero. It may be seen that the radial displacement on both the upper and lower surfaces of the layer move out of phase, putting the centre of the layer in compression and then tension. The radial stress in the layer rises to a maximum at the midpoint before falling to a lower amplitude on the inner layer surface.

The tangential response to this radial forcing is shown in Fig. 7, showing that the compression of the material becomes more important as the thickness of the layer is increased, as this also increases the flexural stiffness of the layer. Since the overall layer is isotropic the same resonant frequencies are found in the radial and tangential directions. When considering a multilayer structure, although each individual layer is isotropic, the structure as a whole is 
orthotropic, with potentially different wave speeds in the axial and circumferential directions to the thickness direction.

\section{$5 \quad$ Tyre parameters}

The parameters used to define the tyre were kindly provided by Dunlop Tyres and included the design data on the material parameters used in the manufacturing stage and their dimensions for a 225/45ZR17 plain tread tyre [17], as shown in Fig. 8. The layers of material are listed in Table 1, from the inside to the outside of the tyre, which include two layers of plies, two breakers, one bandage and an outer rubber layer into which the tread pattern is cut. The bandage contains cords which run in a circumferential direction whereas the plies contain cords which run axially along the belt. The breaker layers contain steel cords, laid at an angle of $\pm 21^{\circ}$ to the circumferential direction. For the purposes of the analysis in this section, the outer rubber layer is not included, hence the characteristic vibrations refer only to the tyre belt. The parameters of the thin fabric layers were estimated using data provided by Dunlop Tyres to determine an equivalent Young's modulus and damping coefficient.

The material damping is incorporated as a complex Young's modulus, assuming a temperature of $20^{\circ} \mathrm{C}$. Cremer, Heckl and Ungar [23] have tabulated loss factors for various materials, referenced with Young's modulus and density. It is recognised that the loss factors for the rubber are highly dependent on temperature and since the outer rubber layer runs in contact with the road surface, this layer will convert energy from the movement and friction between the rubber and road surface into heat. This temperature increase also depends on the vehicle speed, cornering, braking and accelerating manoeu- 
vres and is time dependent, however, this paper considers the temperature to be at a steady state. Hence, the effect of this additional heat increase may be incorporated in the model through changes to the material properties and the amount of damping applied through the complex Young's modulus. The damping factors used in this model vary from low amplitudes for steel layers (0.0002) and fabric layers (0.015) to large amplitudes for certain rubber layers (0.4), with all damping assumed to remain constant with frequency.

\section{Results and discussion}

The response of the multilayer tyre belt is more complicated than for a single viscoelastic layer as it is possible for resonances to occur in each individual layer as well as a coupled system of masses on springs. It is possible for two or more layers to resonate at different frequencies, which may cause vibration motion to be damped or accentuated in other layers.

The point mobility of the tyre belt in the radial direction, defined as the ratio of the radial velocity resulting from a radial force input $\tilde{v}_{r} / \tilde{\sigma}_{r r}\left(r, \omega, n, k_{z}\right)$ is shown in Fig. 9 where the amplitude of the mobility is represented by contours for different frequencies, angular orders and discrete axial mode numbers, $k_{z}=$ $m \pi / w, m=0,1,6,7$.

The vibration modes are consistent with those seen in the single layer, with a compression mode of the belt being dominated by the flexural mode. The resonances due to the air cavity and spring are smaller than the amplitude of the flexural mode yet are important for low angular orders and axial wavenumbers. For higher axial modes, the interaction between the layers with the steel 
cords and the outer rubber layer causes the resonant frequency to rise for low angular orders.

The dominant vibration mode may be verified by an examination of the variations of radial displacement and stress through the belt, by taking a fixed value of angular order $n=15$ and zero axial wavenumber. The flexural mode of vibration is then located at a frequency around $f=700 \mathrm{~Hz}$. The variation in radial displacement, shown in Fig. 10(a), is approximately constant through the thickness of the belt, showing that all radial elements of the belt move together in phase. The radial stress in the belt, Fig. 10(b), takes the value of the boundary condition on the outer surface, decreasing slightly until the layers with the steel cords have been passed and then increasing rapidly to the large value of stress on the inside surface of the belt, where the air cavity and springs provide a restoring force to motion.

The inverse Fourier transform stated in Eq. (6) may now be applied in order to find the radial displacement of the tyre belt as a function of spatial coordinates rather than wavenumbers. The inversion from $k_{z}$ to $z$ is performed by completing the Fourier sum of the mode numbers $m$, which applies a periodic boundary condition at $z= \pm w / 2$. It is assumed that for the purposes of analysing the modal response of the tyre belt for external noise radiation, that the sidewalls do not deform either radially, tangentially or axially (this is a simplification, however it allows the characteristics of different belt materials to be analysed and compared easily). The inversion sum from angular order to circumferential angle $n$ to $\theta$ is also performed and is shown in Fig. 11(a) as $\tilde{u}_{r} / \tilde{\sigma}_{r r}(r, \omega, \theta, z)$ for $z=0 \mathrm{~m}$. Above $500 \mathrm{~Hz}$ the vibrations resulting from the applied forcing at $\theta=0$ are not seen around the whole circumference of the tyre, due to the large damping present in the materials. The vibration is atten- 
uated significantly for increasing frequencies and above $2 \mathrm{kHz}$, the resonances are localised to the forcing location.

The inversion from frequency to the time domain is shown in Fig. 11(b) as contours of $u_{r} / \sigma_{r r}(r, t, \theta, z)$ for $z=0 \mathrm{~m}$. The dominant flexural wave speed, which from the simplified analysis carried out in Eq. (36) is a function of the wavenumber, is in the range 50 to $65 \mathrm{~m} / \mathrm{s}$, with a faster compression wave propagating at around $155 \mathrm{~m} / \mathrm{s}$.

The addition of a thick layer of tread rubber onto the surface of the belt increases the amount of damping in the structure and modifies the resonant frequencies of the dominant flexural wave for the point mobility in the radial direction for the axial mode $m=0, \tilde{v}_{r} / \tilde{\sigma}_{r r}\left(r, \omega, n, k_{z}\right)$ as shown in Fig. 12(a). This dominant dispersive wave is slightly slower than just with the belt alone. This may be confirmed by looking at the completed inversion, $\tilde{u}_{r} / \tilde{\sigma}_{r r}(r, t, \theta, z)$ for $z=0 \mathrm{~m}$ in Fig 12(b). Many of the materials in the tyre belt have a Poisson's ratio close to incompressible (typically 0.45 ), such that when a radial stress is applied a smaller displacement tangential to the surface occurs.

Results for tangential forcing are shown in Figs 13 to 15, where now the radial force on the outer cylindrical surface is zero. The point mobility relating the ratio of the tangential component of velocity to the tangential component of applied stress is shown in Fig. 13, which is important for the noise generated when a vehicle is accelerating or braking. These resonant frequencies are typically higher than those for the radial forcing, reaching a cut on frequency in excess of $2 \mathrm{kHz}$ for the fifth axial mode across the tyre width and are dominated by compression waves propagating in the circumferential directions. Due to the incompressibility of a number of the layers of the tyre belt, this 
tangential shear stress also excites radial flexural and compression modes in different layers, which appear as weak resonances. The peak amplitude of the dominant compression resonance is approximately twice the peak amplitude of the radial velocity when radially excited.

The inverse Fourier transform is now applied to the results for the tangential displacement which occurs with tangential forcing. The displacement response as a function of axial position and frequency, for zero angular order, is shown in Fig. 14(a), $\tilde{u}_{\theta} / \tilde{\sigma}_{r \theta}(r, \omega, n, z)$. For a given axial vibration mode, the cut on frequency may be observed, in addition to the antinodes across the tyre width. Five vibration modes are visible below the $2.5 \mathrm{kHz}$ acoustic range of interest, however significant amplitude is still present at the forcing location, $z=0$ for frequencies up to $4.5 \mathrm{kHz}$.

The response against angular order is also shown, $\tilde{u}_{\theta} / \tilde{\sigma}_{r \theta}(r, \omega, n, z)$ for the case of $z=0 \mathrm{~m}$. These results indicate that when considering the vibrations due to acceleration and braking forces on the tyre, that angular orders up to $n=50$ should be considered, despite the high levels of damping present in several layers of materials.

The inverse Fourier transform from angular order to the angle around the tyre $\theta$ is now considered. As with the inversion over axial mode number, this takes the form of a Fourier sum. The tangential displacement of the tyre belt as a function of $\theta$ when excited tangentially is shown in Fig. 15(a) as $\tilde{u}_{\theta} / \tilde{\sigma}_{r \theta}(r, \omega, \theta, z)$. It may be seen that up to a frequency of $f=1.5 \mathrm{kHz}$, the response is modal around the tyre, however above approximately $2.7 \mathrm{kHz}$ the resonance is limited to a small area around the forcing position $\theta=0$. In the time domain, $u_{\theta} / \sigma_{r \theta}(r, t, \theta, z=0)$ in Fig. 15(b) these modal resonances are 
seen to propagate from the bottom of the tyre $\theta=0$ to the top $\theta=\pi$ rads and return back, all of the time attenuating due to the large damping in the tyre belt materials. The speed of the dominant compression wave is estimated to be approximately $150 \mathrm{~m} / \mathrm{s}$, significantly faster than the flexural wave speed.

\section{Conclusions}

In order to determine the noise produced by a vehicle tyre rolling on a rough road surface, the vibration characteristics of a stationary tyre must be determined and understood. A description has been provided in this paper for a model of the tyre belt, which can predict the vibration characteristics, including resonant frequencies, mode shapes and wave speeds using a multilayer cylindrical viscoelastic structure whose parameters are defined solely by design data without the requirement for experimental testing. This model, which includes the effects of curvature, can provide the displacement or velocity response of the tyre belt when excited in the radial or tangential directions by a force. Each layer of the tyre belt is considered to be isotropic in the circumferential and axial directions, however the composite structure is orthotropic in the radial direction. The behaviour of the displacements and stresses at any point through the thickness of the multilayer structure may be obtained either as functions of frequency, mode numbers or as functions of spatial coordinates.

This model takes into account the sidewalls by assuming the sides of the tyre belt are pinned and making the tyre belt periodic in the axial direction. The model also takes into account the air cavity and wheel hub for a range of excitation frequencies and mode shapes. 
Using a single thick cylindrical layer of rubber, the two primary resonant waves which are excited by an applied radial force are shown to be a dispersive flexural wave and a faster non-dispersive compression wave. For a multilayer structure such as a tyre belt, resonances may occur in individual layers which may accentuate or damp vibration modes of the whole structure. When excited by an applied radial load, the response of the flexural wave dominates the vibration amplitude due to the compression wave or the resonance from the air cavity. This dominant flexural wave travels at an approximate speed of between 50 and $65 \mathrm{~m} / \mathrm{s}$ in the tyre geometry.

When the tyre belt is excited in the tangential direction, the radial velocity in the tangential direction has a compression wave which travels at a speed of approximately $150 \mathrm{~m} / \mathrm{s}$. The amplitude of these resonances extend up to a frequency of approximately $4 \mathrm{kHz}$ and are important for the noise generated by the tyre as it undergoes accelerating or braking manoeuvres.

The response of the tyre belt has been examined for a range of frequencies with results displayed in both the frequency-wavenumber and time-spatial domains. The model and the results are suitable for use in a separate numerical program which can be used to predict the exterior noise generated by a patterned tyre rolling on a rough road surface. The results from this paper will be used in order to obtain a simple bending plate model of the tyre response, which includes a more accurate representation of the tyre sidewalls. The work carried out in this paper is therefore an important step in the prediction of tyre noise from the tyre design phase. 


\section{Acknowledgements}

My appreciation to the Engineering and Physical Sciences Research Council (EPSRC) for funding this work, as well as Goodyear Dunlop tyres, LandRover and TRL for providing facilities, experimental results and industrial information on tyre dynamics and design. 


\section{Appendix A: Displacement and stress in a visco-elastic layer}

The three components of displacement on the surface of an elastic layer are written in terms of the scalar and vector potentials once the Fourier transform has been applied,

$$
\begin{aligned}
& \tilde{u}_{r}=\frac{\partial \tilde{\phi}}{\partial r}+\frac{\mathrm{i} n}{r} \tilde{A}_{z}-\mathrm{i} k_{z} \tilde{A}_{\theta}, \\
& \tilde{u}_{\theta}=\tilde{\phi} \frac{\mathrm{i} n}{r}+k_{z} \mathrm{i} \tilde{A}_{r}-\frac{\partial \tilde{A}_{z}}{\partial r}, \\
& \tilde{u}_{z}=\tilde{\phi} \mathrm{i} k_{z}+\frac{\partial \tilde{A}_{\theta}}{\partial r}+\frac{1}{r} \tilde{A}_{\theta}-\frac{\mathrm{i} n}{r} \tilde{A}_{r} .
\end{aligned}
$$

The three components of traction on the surface of an elastic layer are written in terms of the scalar and vector potentials once the Fourier transform has been applied,

$$
\begin{aligned}
\tilde{\sigma}_{r r}= & -(\lambda+2 \mu)\left(\frac{1}{r} \frac{\partial \tilde{\phi}}{\partial r}+\left(\gamma^{2}-\frac{n^{2}}{r^{2}}\right) \tilde{\phi}\right)+ \\
& 2 \mu\left(-\frac{\mathrm{i} n}{r^{2}} \tilde{A}_{z}+\frac{\mathrm{i} n}{r^{2}} \frac{\partial \tilde{A}_{z}}{\partial r}-\mathrm{i} k_{z} \frac{\partial \tilde{A}_{\theta}}{\partial r}\right)+\lambda\left(\frac{1}{r} \frac{\partial \tilde{\phi}}{\partial r}-\frac{n^{2}}{r^{2}} \tilde{\phi}-k_{z}^{2} \tilde{\phi}\right), \\
\tilde{\sigma}_{r \theta}= & -\tilde{\phi}\left(\frac{2 \mathrm{i} n \mu}{r^{2}}\right)+\frac{\partial \tilde{\phi}}{\partial r}\left(\frac{2 \mathrm{i} n \mu}{r}\right)-\mathrm{i} \tilde{A}_{r}\left(\frac{\mu k_{z}}{r}\right)+\mathrm{i} \frac{\partial \tilde{A}_{r}}{\partial r}\left(\mu k_{z}\right) \\
& +\tilde{A}_{\theta}\left(\frac{\mu n k_{z}}{r}\right)+\tilde{A}_{z}\left(\mu \eta^{2}-\frac{2 n^{2} \mu}{r^{2}}\right)+\frac{\partial \tilde{A}_{z}}{\partial r}\left(\frac{2 \mu}{r}\right), \\
\tilde{\sigma}_{r z}= & \frac{\partial \tilde{\phi}}{\partial r}\left(2 \mathrm{i} k_{z} \mu\right)-\mathrm{i} \tilde{A}_{r}\left(\frac{n \mu}{r^{2}}\right)-\mathrm{i} \frac{\partial \tilde{A}_{r}}{\partial r}\left(\frac{n \mu}{r}\right)+ \\
& \tilde{A}_{\theta}\left(k_{z}^{2} \mu-\eta^{2} \mu+\frac{n^{2} \mu}{r^{2}}\right)-\tilde{A}_{z}\left(\frac{n \mu k_{z}}{r}\right) .
\end{aligned}
$$

The coefficients used to determine the components of stress and displacement in the final form of the viscoelastic cylindrical tyre belt are provided. The formula defining the different matrices is shown, with the displacement matrix, $\mathbf{H}$ and the matrix containing the terms relating to stress $\mathbf{G}$, both six by three 
in size. The matrix containing the terms with the Wronskians $\mathbf{W}$ and the matrix used to provide a summation method $\mathbf{S}$ are both six by six.

$$
\left(\begin{array}{c}
\tilde{\sigma} \\
\tilde{\mathbf{u}}
\end{array}\right)=\left(\begin{array}{c}
\mathrm{G} \\
\mathbf{H}
\end{array}\right) \text { W S C. }
$$

The matrix $\mathbf{C}$ contains the constants for the solutions to the wave equation in terms of the scalar and vector potentials as $c_{1}, c_{2}, c_{3}, c_{4}, c_{7}, c_{8}$. The constants $c_{5}, c_{6}$ have been eliminated as the divergence of the vector potential is zero.

$$
\begin{aligned}
\tilde{\phi}(\gamma r) & =c_{1} J_{n}(\gamma r)+c_{2} Y_{n}(\gamma r), \\
\left(\mathrm{i} \tilde{A}_{r}-\tilde{A}_{\theta}\right) & =2 c_{3} J_{(n+1)}(\eta r)+2 c_{4} Y_{(n+1)}(\eta r), \\
\left(\mathrm{i} \tilde{A}_{r}+\tilde{A}_{\theta}\right) & =2 c_{5} J_{(n-1)}(\eta r)+2 c_{6} Y_{(n-1)}(\eta r), \\
\tilde{A}_{z} & =c_{7} J_{n}(\eta r)+c_{8} Y_{n}(\eta r) .
\end{aligned}
$$

The coefficients of the matrix for the stress terms $G_{i j}$ are,

$$
\begin{aligned}
\Gamma_{1} & =\left(\frac{J_{n+1}(\gamma r)}{J_{n}(\gamma r)}+\frac{Y_{n+1}(\gamma r)}{Y_{n}(\gamma r)}\right), \\
\Gamma_{2} & =\left(\frac{J_{n+1}(\eta r)}{J_{n}(\eta r)}+\frac{Y_{n+1}(\eta r)}{Y_{n}(\eta r)}\right), \\
\Gamma_{3} & =\left(\frac{J_{n}(\eta r)}{J_{n+1}(\eta r)}+\frac{Y_{n}(\eta r)}{Y_{n+1}(\eta r)}\right) .
\end{aligned}
$$

$$
\begin{aligned}
G_{11}= & -2 \gamma^{2}(\lambda+2 \mu)+4 \mu n(n-1) / r^{2}-2 k_{z}^{2} \lambda+(2 \mu \gamma / r) \Gamma_{1}, \\
G_{12}= & 2 \mu \gamma / r \\
G_{13}= & -8 \mathrm{i} k_{z} \mu / r+\left(-4 n(n-1) \mathrm{i} k_{z} \mu /\left(\eta r^{2}\right)+4 \eta \mathrm{i} k_{z} \mu\right) \Gamma_{3}, \\
G_{14}= & -4 n(n-1) \mathrm{i} k_{z} \mu /\left(\eta r^{2}\right)+4 \eta \mathrm{i} k_{z} \mu, \\
G_{15}= & 8 n(n-1) \mathrm{i} k_{z}^{2} \mu /(\eta r)^{2}-4 \mathrm{i} k_{z}^{2} \mu-4 \mu \mathrm{i} n / r^{2}+4 \mathrm{i} n^{2} \mu / r^{2}+ \\
& \left(-2(n-1) k_{z}^{2} \mu \mathrm{i} /(\eta r)-2 \mathrm{i} n \eta \mu / r\right) \Gamma_{2}, \\
G_{16}= & \left(-2(n-1) k_{z}^{2} \mu \mathrm{i} /(\eta r)-2 \mathrm{i} n \eta \mu / r\right) .
\end{aligned}
$$




$$
\begin{aligned}
G_{21}= & 4 \mathrm{i} n \mu(n-1) / r^{2}-2 \mathrm{i} n \mu \gamma / r \Gamma_{1}, \\
G_{22}= & -2 \mathrm{i} n \mu \gamma / r, \\
G_{23}= & -8 n \mu k_{z} / r+4 n(n-1) \mu k_{z} /\left(\eta r^{2}\right) \Gamma_{3}, \\
G_{24}= & 4 n(n-1) \mu k_{z} /\left(\eta r^{2}\right), \\
G_{25}= & 2 \mu k_{z}^{2}+2 \eta^{2} \mu-4 n \mu(n-1) / r^{2}-8 n(n-1) k_{z}^{2} \mu /\left(\eta r^{2}\right)+ \\
& \left(2(n-1) k_{z}^{2} \mu /(\eta r)-2 \mu \eta / r\right) \Gamma_{2}, \\
G_{26}= & 2(n-1) k_{z}^{2} \mu /(\eta r)-2 \mu \eta / r .
\end{aligned}
$$

$$
\begin{aligned}
& G_{31}=4 \mathrm{i} k_{z} \mu n / r-2 \mathrm{i} k_{z} \mu \gamma \Gamma_{1}, \\
& G_{32}=-2 \mathrm{i} k_{z} \mu \gamma, \\
& G_{33}=4 \mu\left(\eta^{2}-k_{z}^{2}\right)+2 n \mu /(\eta r)\left(k_{z}^{2}-\eta^{2}\right) \Gamma_{3}, \\
& G_{34}=2 n \mu /(\eta r)\left(k_{z}^{2}-\eta^{2}\right), \\
& G_{35}=-4 n k_{z}^{3} \mu /\left(\eta^{2} r\right)+\left(k_{z}^{3} \mu / \eta-\mu k_{z} \eta\right) \Gamma_{2}, \\
& G_{36}=k_{z}^{3} \mu / \eta-\mu k_{z} \eta .
\end{aligned}
$$

The coefficients of the matrix for displacement $H_{i j}$, using the same notation as in the previous matrix, are,

$$
\begin{aligned}
& H_{11}=(2 n / r)-\gamma \Gamma_{1}, \\
& H_{12}=-\gamma, \\
& H_{13}=4 \mathrm{i} k_{z}-2 n \mathrm{i} k_{z} /(\eta r) \Gamma_{3}, \\
& H_{14}=-2 n \mathrm{i} k_{z} /(\eta r), \\
& H_{15}=2 \mathrm{i} n / r+4 n k_{z}^{2} \mathrm{i} /\left(\eta^{2} r\right)-\mathrm{i} k_{z}^{2} / \eta \Gamma_{2}, \\
& H_{16}=-\mathrm{i} k_{z}^{2} / \eta .
\end{aligned}
$$

$$
\begin{aligned}
& H_{21}=2 \mathrm{i} n / r, \\
& H_{22}=0, \\
& H_{23}=2 n k_{z} /(\eta r) \Gamma_{3}, \\
& H_{24}=2 n k_{z} /(\eta r), \\
& H_{25}=\left(k_{z}^{2} / \eta+\eta\right) \Gamma_{2}-\left(4 n k_{z}^{2}+2 n \eta^{2}\right) /\left(\eta^{2} r\right), \\
& H_{26}=k_{z}^{2} / \eta+\eta .
\end{aligned}
$$




$$
\begin{aligned}
& H_{31}=2 \mathrm{i} k_{z}, \\
& H_{32}=0, \\
& H_{33}=-2 \eta \Gamma_{3}, \\
& H_{34}=-2 \eta, \\
& H_{35}=2 k_{z}, \\
& H_{36}=0 .
\end{aligned}
$$

$$
\mathbf{W}=\left(\begin{array}{cccccc}
1 & 0 & 0 & 0 & 0 & 0 \\
0 \frac{2}{\pi \gamma r J_{n}(\gamma r) Y_{n}(\gamma r)} & 0 & 0 & 0 & 0 \\
0 & 0 & 1 & 0 & 0 & 0 \\
0 & 0 & 0-\frac{2}{\pi \eta r J_{n+1}(\eta r) Y_{n+1}(\eta r)} & 0 \\
0 & 0 & 0 & 0 & 1 & 0 \\
0 & 0 & 0 & 0 & 0 & \frac{2}{\pi \eta r J_{n}(\eta r) Y_{n}(\eta r)}
\end{array}\right) .
$$

$$
\mathbf{S}=\left(\begin{array}{cccccc}
1 / 2 & 1 / 2 & 0 & 0 & 0 & 0 \\
1 / 2 & -1 / 2 & 0 & 0 & 0 & 0 \\
0 & 0 & 1 / 2 & 1 / 2 & 0 & 0 \\
0 & 0 & 1 / 2 & -1 / 2 & 0 & 0 \\
0 & 0 & 0 & 0 & 1 / 2 & 1 / 2 \\
0 & 0 & 0 & 0 & 1 / 2 & -1 / 2
\end{array}\right)
$$


The scaling matrix $\mathbf{M}$, is given as,

$$
\mathbf{M}=\left(\begin{array}{cccccc}
\frac{J_{n}\left(\gamma r_{j+1}\right)}{J_{n}\left(\gamma r_{j}\right)} & 0 & 0 & 0 & 0 & 0 \\
0 & \frac{Y_{n}\left(\gamma r_{j+1}\right)}{Y_{n}\left(\gamma r_{j}\right)} & 0 & 0 & 0 & 0 \\
0 & 0 & \frac{J_{n+1}\left(\gamma r_{j+1}\right)}{J_{n+1}\left(\gamma r_{j}\right)} & 0 & 0 & 0 \\
0 & 0 & 0 & \frac{Y_{n+1}\left(\gamma r_{j+1}\right)}{Y_{n+1}\left(\gamma r_{j}\right)} & 0 & 0 \\
0 & 0 & 0 & 0 & \frac{J_{n}\left(\gamma r_{j+1}\right)}{J_{n}\left(\gamma r_{j}\right)} & 0 \\
0 & 0 & 0 & 0 & 0 & \frac{Y_{n}\left(\gamma r_{j+1}\right)}{Y_{n}\left(\gamma r_{j}\right)}
\end{array}\right) .
$$

\section{References}

[1] Future noise policy, COM(96)540, European Commission, Green Paper (1996).

[2] Transport trends, 2002. National Statistics UK. Transport Statistics Publications, The Stationary Office, London (November 2002).

[3] U. Sandberg, Tyre/road noise - myths and realities (plenary paper), in: Proceedings of the International Congress and Exhibition on Noise Control Engineering, the Netherlands, 2001.

[4] F. Takahashi, G. Watanabe, M. Nakada, H. Sakata, M. Ikeda, Tyre/suspension system modelling for investigation of road noise characteristics, International Journal of Vehicle Design 8 (4-6) (1987) 588-597.

[5] S. Saigal, T. Kim, W. Soedel, Free vibration of a tire as a toroidal membrane, Journal of Sound and Vibration 107 (1) (1986) 71-82. 
[6] A. Pietrzyk, Prediction of the dynamic response of a tire, in: Proceedings of Internoise, 2001.

[7] W. Kropp, Structure-borne sound on a smooth tyre, Applied Acoustics 26 (3) (1989) 181-192.

[8] W. Kropp, A mathematical model of tyre noise generation, Heavy Vehicle Systems, International Journal of Vehicle Design 6 (1-4) (1999) 310-329.

[9] B. Kim, Prediction of sound radiation from tire tread-band vibration, International Journal of Automotive Technology 2 (1) (2001) 33-38.

[10] Y.-J. Kim, S. Bolton, Effect of rotation on the vibration characteristics of tires, in: Proceedings of Internoise, 2002.

[11] S. Huang, The vibration of rolling tyres in ground contact, International Journal of Vehicle Design 13 (1) (1992) 78-95.

[12] R. Keltie, Analytical model of the truck tire vibration sound mechanism, Journal of the Acoustical Society of America 71 (2) (1982) 359-367.

[13] R. Graf, Tyre-road interaction noise, Ph.D. thesis, Department of Engineering, University of Cambridge (2002).

[14] G. Batchelor, An Introduction to Fluid Dynamics, Cambridge University Press, 1967.

[15] P. Morse, H. Feshback, Methods of Theoretical Physics, Part 1, New York, 1953.

[16] A. Armenàkas, D. Gazis, G. Hermann, Free Vibrations of Circular Cylindrical Shells, Pergamon Press, 1969.

[17] D. O'Boy, Tyre-road noise, Ph.D. thesis, Department of Engineering, University of Cambridge (2005).

[18] M. Abramowitz, I. Stegun, Handbook of Mathematical Functions with Formulas, Graphs and Mathematical Tables, Dover, 1972. 
[19] D. Gazis, Three dimensional investigation of the propagation of waves in hollow circular cylinders- i. analytical foundation ii. numerical results, Journal of the Acoustical Society of America 31 (1959) 568-78.

[20] J. Goodier, S. Timoshenko, Theory of Elasticity, McGraw-Hill International Editions, 1970.

[21] G. Lamé, Lecons sur la théorie de l'élasticité, Gauthier-Villars, Paris, 1852.

[22] K. Graff, Wave Motion in Elastic Solids, Clarendon Press, Oxford, 1975.

[23] M. Heckl, L. Cremer, E. Ungar, Structure Borne Sound, 2nd Edition, SpringerVerlag, 1988. 


\begin{tabular}{llll}
\hline Layer number & Name & Thickness $(\mathrm{mm})$ & Cords \\
\hline 1 & Inner rubber & 1.40 & N/A \\
2 & Ply & 1.40 & Fabric \\
3 & Ply & 1.40 & Fabric \\
4 & Breaker & 1.42 & Steel \\
5 & Breaker & 1.42 & Steel \\
6 & & & Fabric \\
7 & Bandage & 1.00 & N/A \\
\hline Table 1 & Outer rubber & 2.50 & N/A \\
\hline
\end{tabular}

Thickness of material layers and any cords present in the tyre cross section.

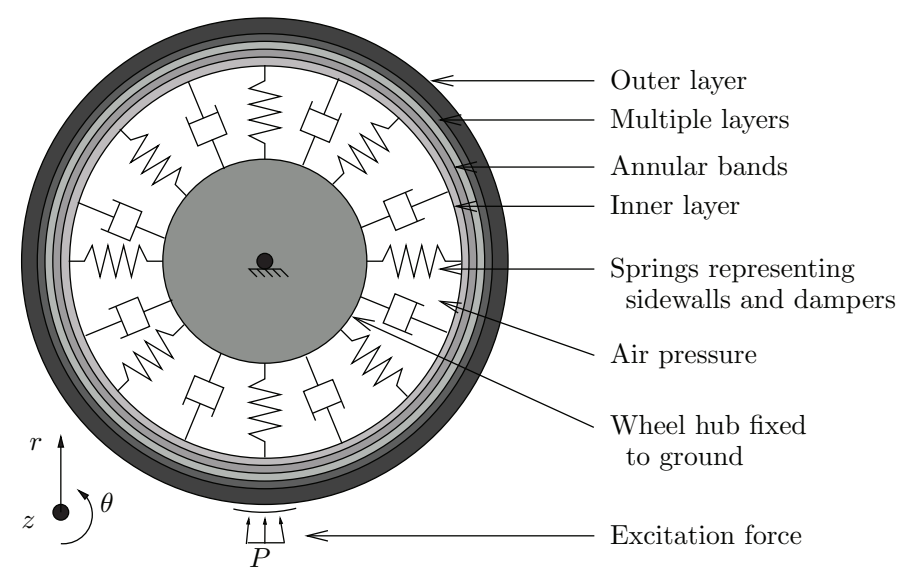

Fig. 1. Complete viscoelastic cylindrical model of the tyre belt. 


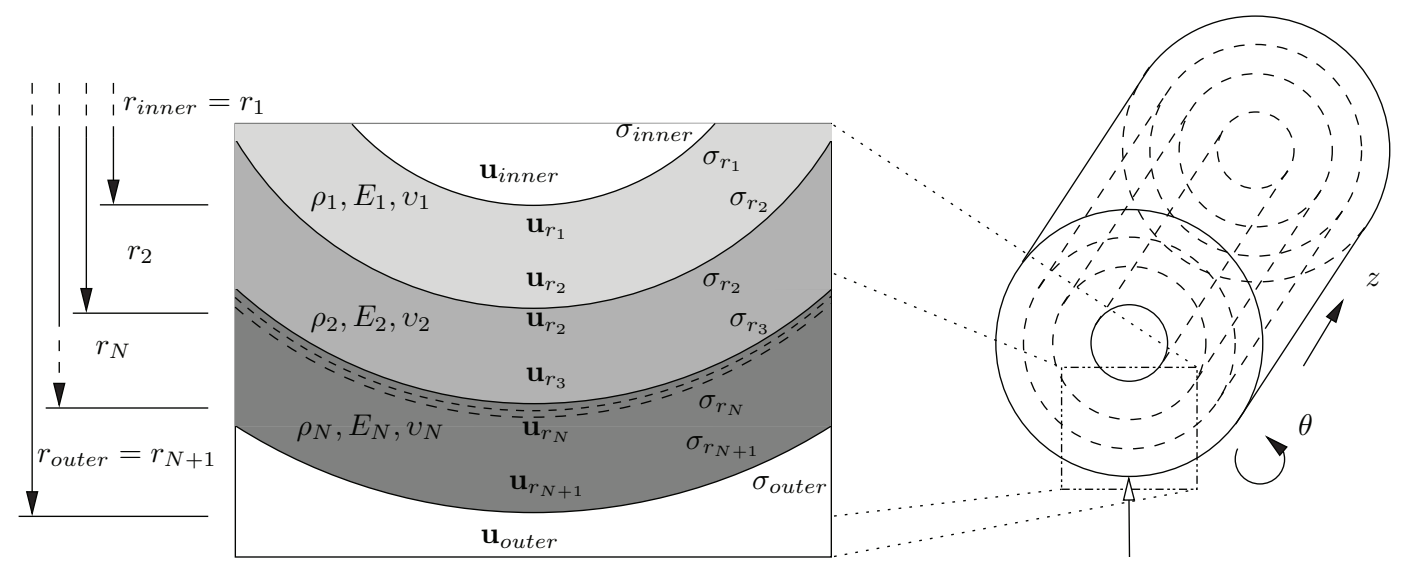

Fig. 2. Illustration of the multiple layers of material laid together, which comprise the tyre belt. Parameters defining the material properties, in addition to the components of stress and displacement in each layer are shown.

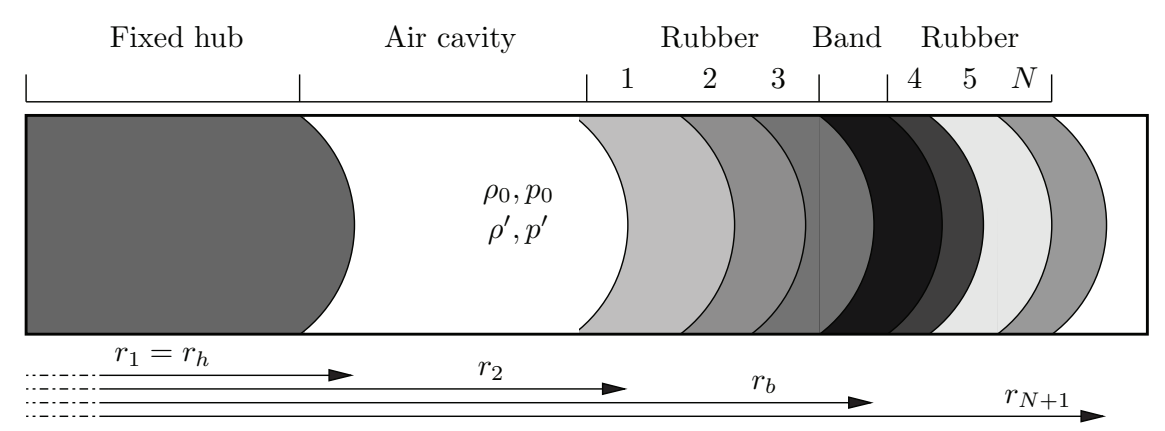

Fig. 3. Cross section of the tyre belt and wheel, showing the inclusion of a pressurised air cavity. This is located between a fixed wheel hub and the multiple layers of material comprising the tyre belt. Also included in the tyre belt are bands of material. 

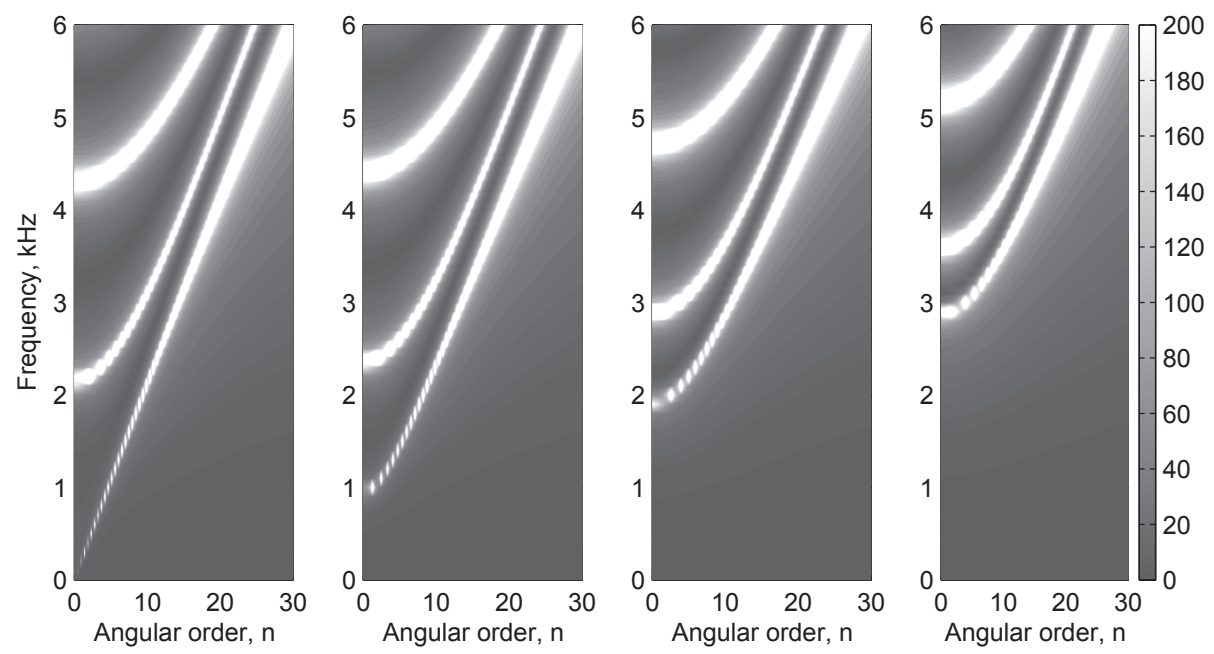

Fig. 4. Effective air stiffness of the air cavity $\left|\tilde{p} / \tilde{u}_{r\left(r=r_{2}\right)}\right|\left[10^{9}\left(\mathrm{~N} / \mathrm{m}^{3}\right)\right]$, for mode numbers in the axial direction $k_{z}=m \pi / w$, where $w$ is the width of the tyre and the axial mode number $m=0,1,2,3$. 


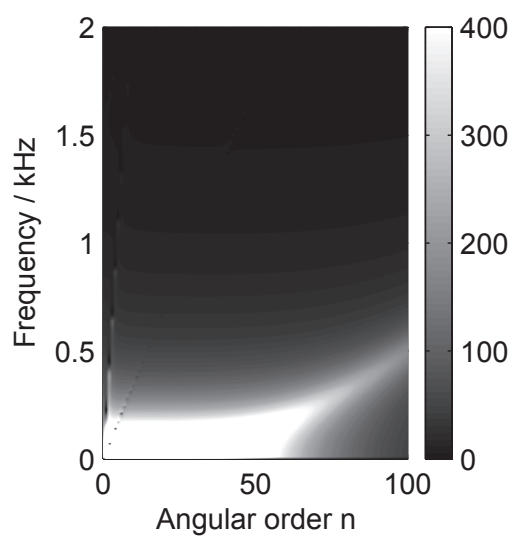

(a)

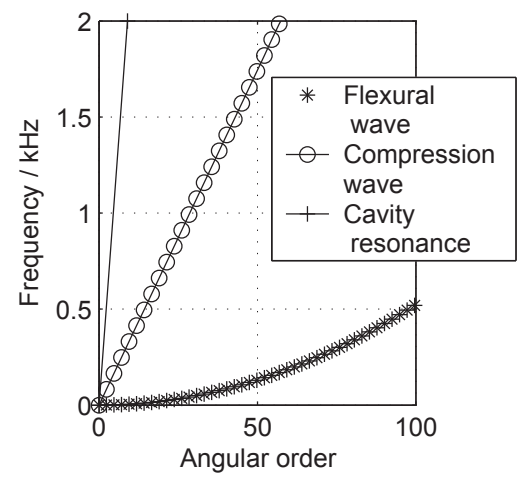

(b)

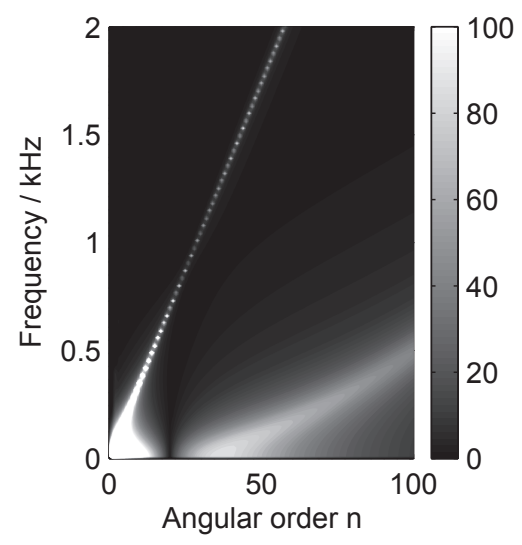

(c)

Fig. 5. Displacement response of a thin elastic layer to radial forcing. (a) Radial displacement $\tilde{u}_{r} / \tilde{\sigma}_{r r}\left(r, \omega, n, k_{z}\right)\left[10^{-9}\left(\mathrm{~m}^{3} / \mathrm{N}\right)\right]$; (b) Locations of the resonant frequencies and the mode type, based on the wave speed analysis of equations (36) and (37); (c) Tangential displacement $\tilde{u}_{\theta} / \tilde{\sigma}_{r r}\left(r, \omega, n, k_{z}\right)\left[10^{-9}\left(\mathrm{~m}^{3} / \mathrm{N}\right)\right]$ 


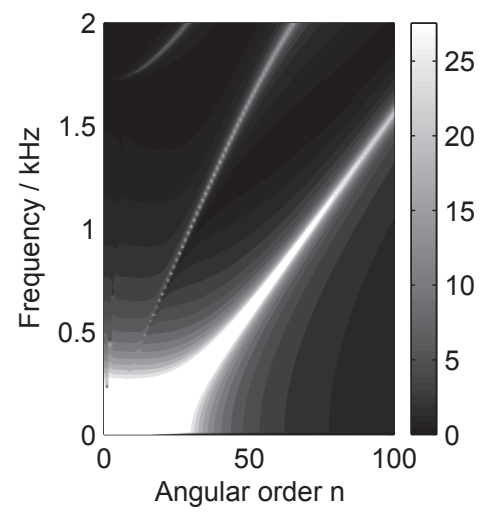

(a)

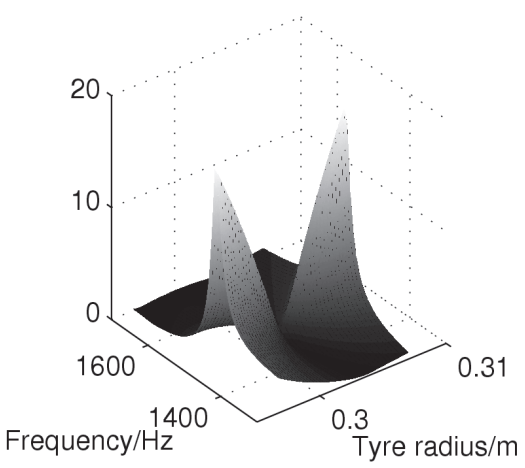

(b)

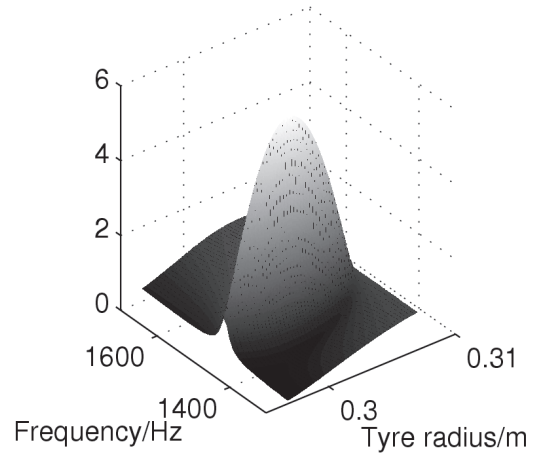

(c)

Fig. 6. Radial response of a single thick layer of rubber material to unit radial forcing, showing a dominant flexural wave and a less significant compressive wave in the material. (a) Radial displacement response $\left|\tilde{u}_{r} / \tilde{\sigma}_{r r}\left(r, \omega, n, k_{z}\right)\right|\left[10^{-9}\left(\mathrm{~m}^{3} / \mathrm{N}\right)\right]$;

(b) Radial displacement through the thickness of the layer $\left|\tilde{u}_{r}\left(r, \omega, n, k_{z}\right)\right|\left[10^{-9}(\mathrm{~m})\right]$;

(c) Radial stress through the thickness of the layer $\left|\tilde{\sigma}_{r r}\left(r, \omega, n, k_{z}\right)\right|\left[10^{-9}\left(\mathrm{~N} / \mathrm{m}^{2}\right)\right]$. 


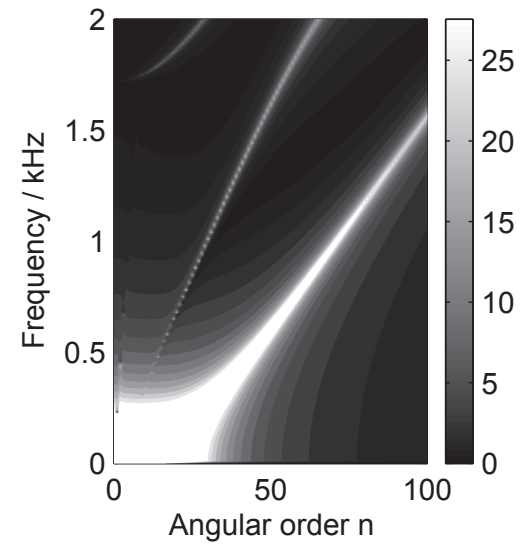

Fig. 7. Tangential response to radial forcing $\left|\tilde{u}_{\theta} / \tilde{\sigma}_{r r}\left(r, \omega, n, k_{z}\right)\right|\left[10^{-9}\left(\mathrm{~m}^{3} / \mathrm{N}\right)\right]$. The amplitude of the compression wave is comparable to that of the flexural wave, as the materials are slightly incompressible. 


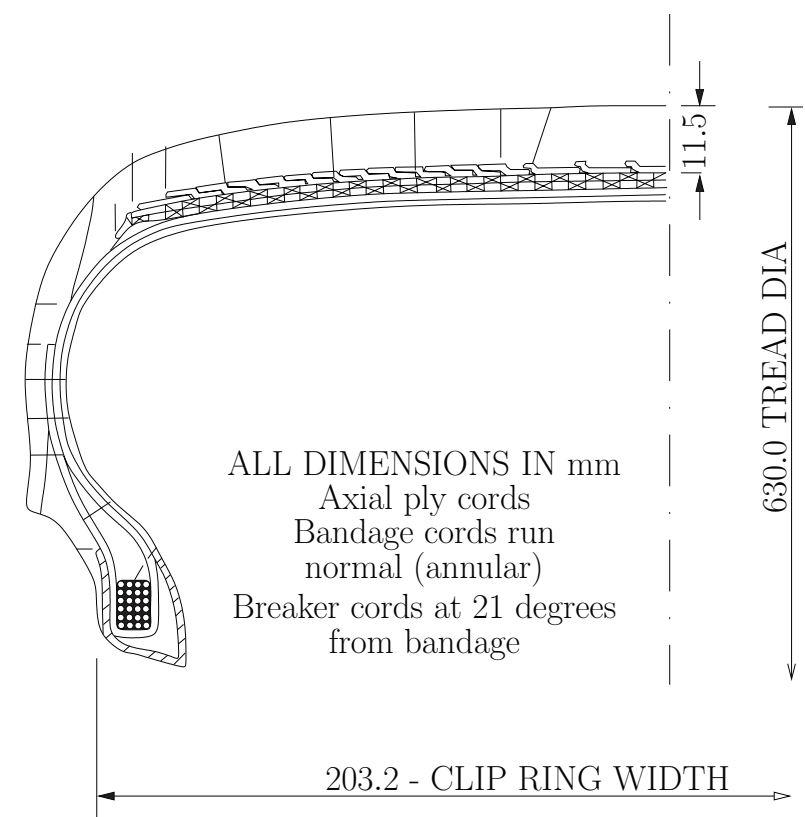

Fig. 8. Cross section of the Dunlop Tyre, of type 225/45ZR17 with a plain tread.

The information used in the design process has been used to define the material properties of each layer of the tyre belt model. 

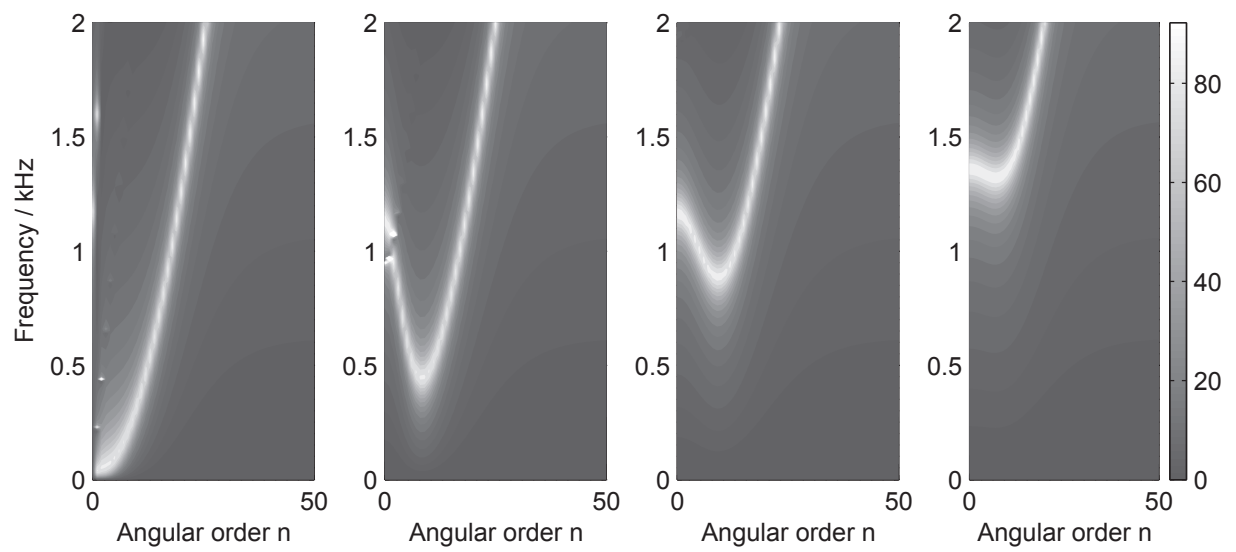

Fig. 9. Point mobility of the tyre belt showing the radial velocity which results from a radial excitation, $\left|\tilde{v}_{r} / \tilde{\sigma}_{r r}\left(r, \omega, n, k_{z}\right)\right|\left[10^{-6}\left(\mathrm{~m}^{3} / \mathrm{N} / \mathrm{s}\right)\right]$. The response at various axial modes numbers are shown, $k_{z}=m \pi / w$ where $m$ is the mode number $m=0,1,6,7$ and $w=0.18 \mathrm{~m}$ is the width of the tyre. 


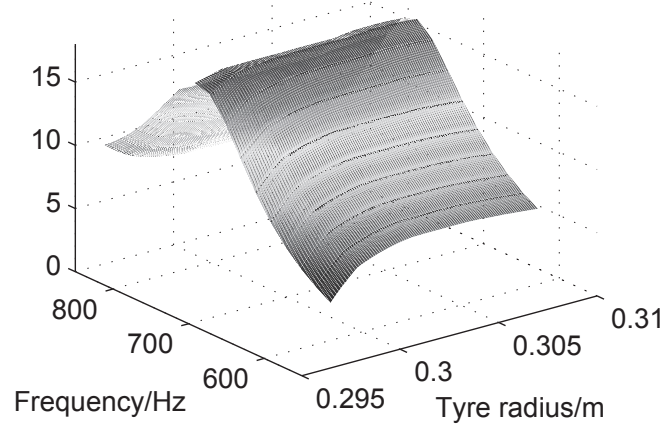

(a)

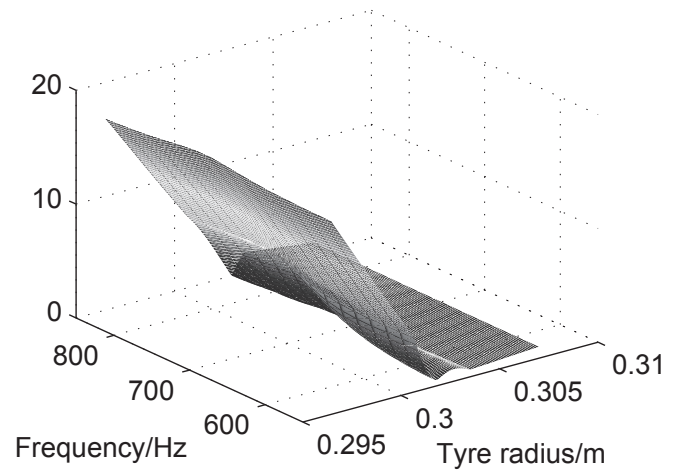

(b)

Fig. 10. Variations in the radial displacement and radial stress in the tyre belt with radius when excited at the resonant frequency for the flexural mode of vibration, angular order $n=15$ and $k_{z}=0 / \mathrm{m}$; (a) The radial displacement $\left|\tilde{u}_{r}\left(r, \omega, n, k_{z}\right)\right|\left[10^{-9}(\mathrm{~m})\right] ;(\mathrm{b})$ The radial stress $\left|\tilde{\sigma}_{r r}\left(r, \omega, n, k_{z}\right)\right|\left[10^{-9}\left(\mathrm{~N} / \mathrm{m}^{2}\right)\right]$. 

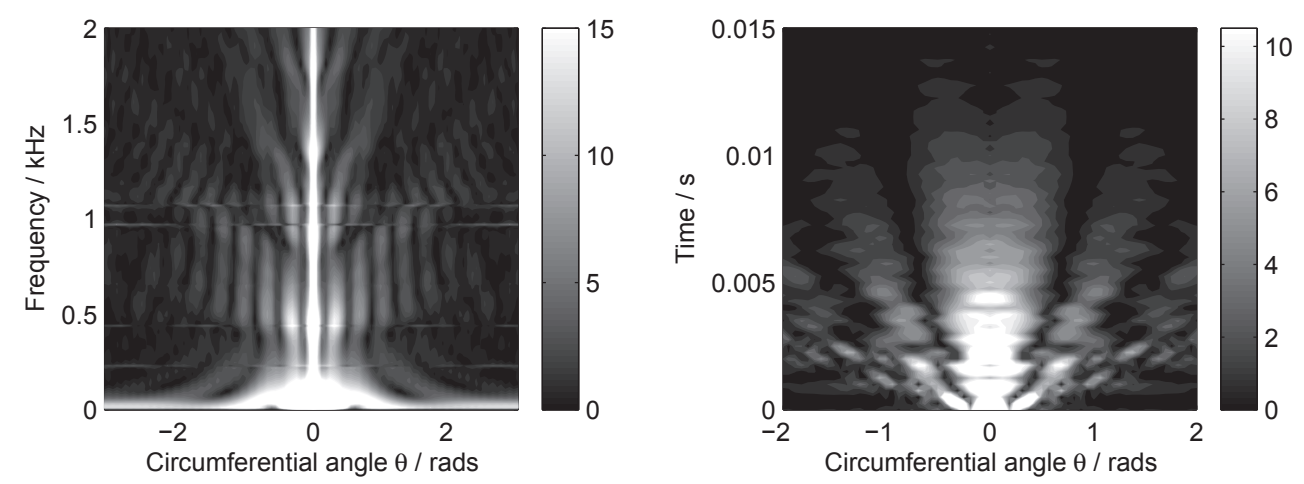

Fig. 11. Radial displacement response of the tyre belt to radial excitation once the Fourier inversions have been completed from the wavenumbers to the spatial domains; (a) Inversion to axial position has been completed, results shown at $z=0 \mathrm{~m}$, $\left|\tilde{u}_{r} / \tilde{\sigma}_{r r}(r, \omega, \theta, z)\right|\left[10^{-9}\left(\mathrm{~m}^{3} / \mathrm{N}\right)\right] ;$ (b) Inversion from frequency to time completed, $\left|u_{r} / \sigma_{r r}(r, t, \theta, z)\right|\left[10^{-6}\left(\mathrm{~m}^{3} / \mathrm{N}\right)\right]$.

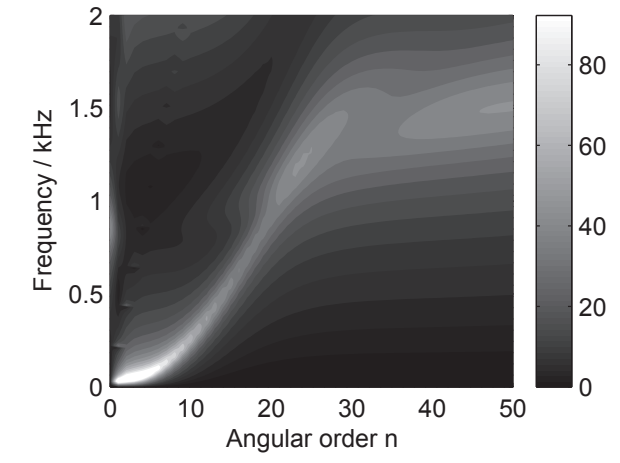

(a)

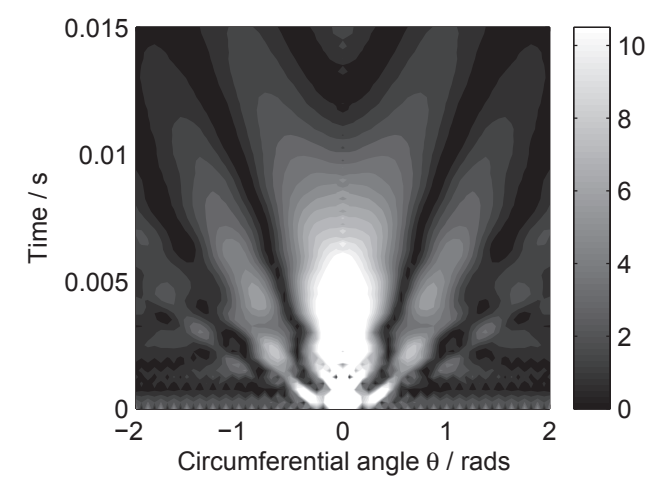

(b)

Fig. 12. Radial response of the tyre belt with a thick layer of tread rubber on the outer surface to radial forcing. (a) Point mobility showing the radial velocity which results from a radial excitation, $\left|\tilde{v}_{r} / \tilde{\sigma}_{r r}\left(r, \omega, n, k_{z}=0\right)\right|\left[10^{-6}\left(\mathrm{~m}^{3} / \mathrm{N} / \mathrm{s}\right)\right]$; (b) Inversion from frequency to time completed, $\left|u_{r} / \sigma_{r r}(r, t, \theta, z=0)\right|\left[10^{-6}\left(\mathrm{~m}^{3} / \mathrm{N}\right)\right]$. 

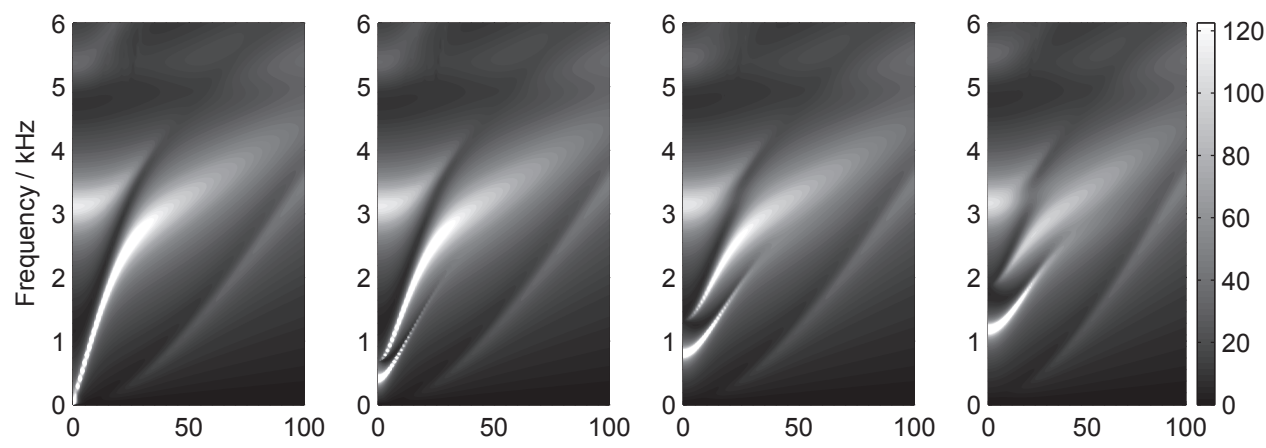

Fig. 13. Point mobility of the tyre belt showing the tangential velocity which results from a tangential excitation, $\left|\tilde{v}_{\theta} / \tilde{\sigma}_{r \theta}\left(r, \omega, n, k_{z}\right)\right|\left[10^{-6}\left(\mathrm{~m}^{3} / \mathrm{N} / \mathrm{s}\right)\right]$. The response at various axial modes numbers are shown, $k_{z}=m \pi / w$ where $m$ is the mode number $m=0,1,2,3$ and $w$ is the width of the tyre.
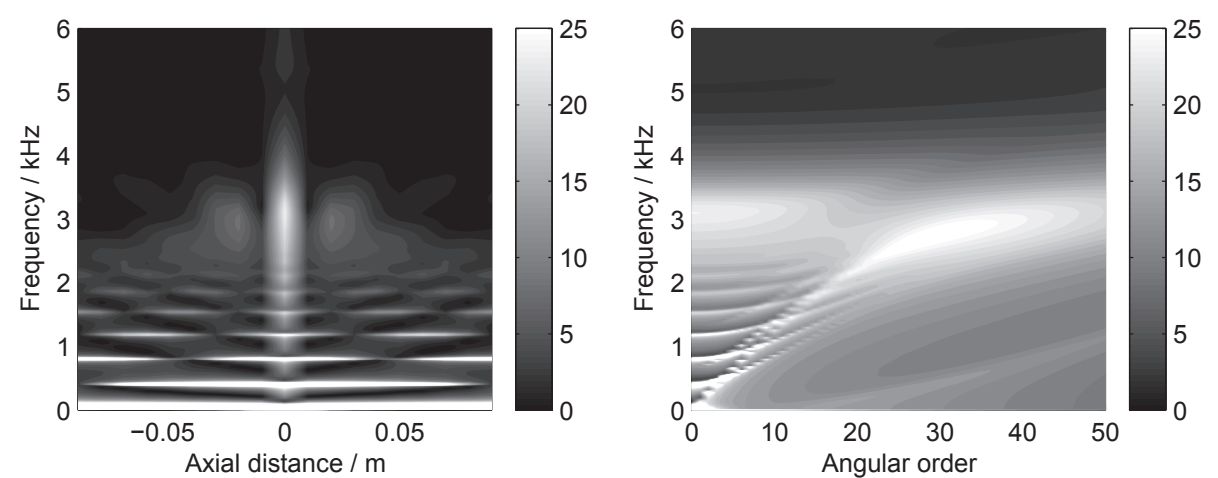

Fig. 14. Tangential displacement of tyre belt to tangential forcing, after the Fourier inversions have been applied. (a) $\left|\tilde{u}_{\theta} / \tilde{\sigma}_{r \theta}(r, \omega, n, z)\right|\left[10^{-9}\left(\mathrm{~m}^{3} / \mathrm{N}\right)\right]$ for an angular order $n=0$. (b) $\left|\tilde{u}_{\theta} / \tilde{\sigma}_{r \theta}(r, \omega, n, z)\right|\left[10^{-9}\left(\mathrm{~m}^{3} / \mathrm{N}\right)\right]$ for an axial position $z=0 \mathrm{~m}$. 

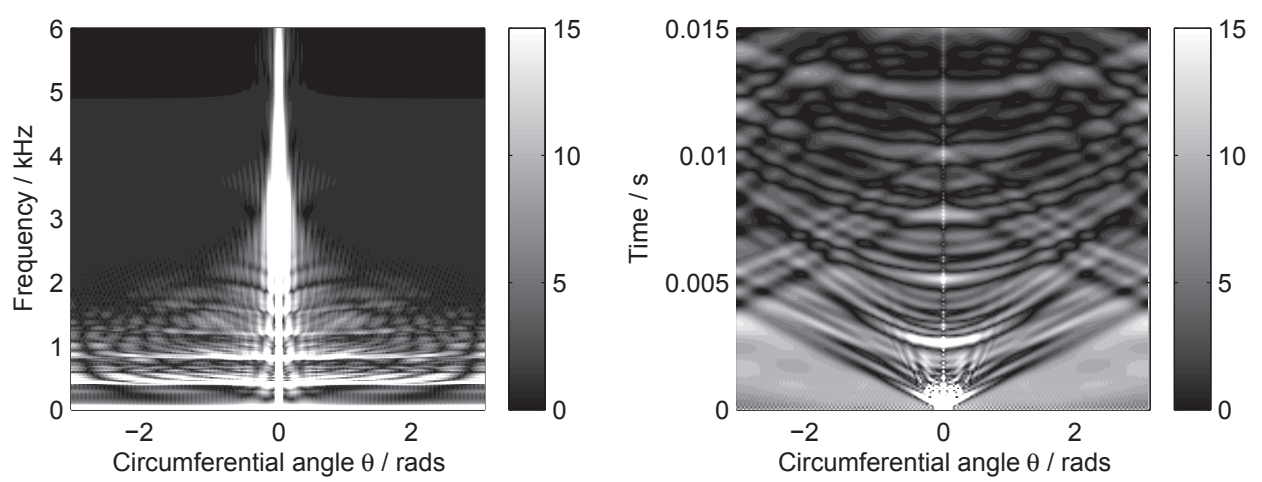

Fig. 15. Tangential displacement of tyre belt to tangential forcing, after the Fourier inversions have been applied. (a) $\left|\tilde{u}_{\theta} / \tilde{\sigma}_{r \theta}(r, \omega, \theta, z)\right|$ for an axial position $z=0 \mathrm{~m}$. (b) $\left|u_{\theta} / \sigma_{r \theta}(r, t, \theta, z)\right|$ for an axial position $z=0 \mathrm{~m}$. 